\title{
Anterior insular cortex mediates hyperalgesia induced by chronic pancreatitis in rats
}

\author{
Yang Bai ${ }^{1+}$, Li-Tian $\mathrm{Ma}^{2 \dagger}$, Yan-Bing Chen ${ }^{3 \dagger}$, Dan Ren ${ }^{4}$, Ying-Biao Chen ${ }^{5}$, Ying-Qi Li ${ }^{6}$, Hong-Ke Sun ${ }^{6}$, Xin-Tong Qiu', \\ Ting Zhang ${ }^{1}$, Ming-Ming Zhang ${ }^{1}$, Xi-Nan Yi ${ }^{7}$, Tao Chen ${ }^{1}$, Hui Li', Bo-Yuan Fan ${ }^{6 *}$ and Yun-Qing $\mathrm{Li}^{1,7^{*}}$
}

\begin{abstract}
Central sensitization plays a pivotal role in the maintenance of chronic pain induced by chronic pancreatitis $(C P)$, but cortical modulation of painful $C P$ remains elusive. This study was designed to examine the role of anterior insular cortex (alC) in the pathogenesis of hyperalgesia in a rat model of CP. CP was induced by intraductal administration of trinitrobenzene sulfonic acid (TNBS). Abdomen hyperalgesia and anxiety were assessed by von Frey filament and open field tests, respectively. Two weeks after surgery, the activation of alC was indicated by FOS immunohistochemical staining and electrophysiological recordings. Expressions of VGluT1, NMDAR subunit NR2B and AMPAR subunit GluR1 were analyzed by immunoblottings. The regulatory roles of alC in hyperalgesia and pain-related anxiety were detected via pharmacological approach and chemogenetics in CP rats. Our results showed that TNBS treatment resulted in long-term hyperalgesia and anxiety-like behavior in rats. CP rats exhibited increased FOS expression and potentiated excitatory synaptic transmission within alC. CP rats also showed up-regulated expression of VGluT1, and increased membrane trafficking and phosphorylation of NR2B and GluR1 within alC. Blocking excitatory synaptic transmission significantly attenuated abdomen mechanical hyperalgesia. Specifically inhibiting the excitability of insular pyramidal cells reduced both abdomen hyperalgesia and pain-related anxiety. In conclusion, our findings emphasize a key role for alC in hyperalgesia and anxiety of painful CP, providing a novel insight into cortical modulation of painful CP and shedding light on alC as a potential target for neuromodulation interventions in the treatment of CP.
\end{abstract}

Keywords: Rat, Chronic pancreatitis, Anterior insular cortex, Long-term potentiation, Excitatory synaptic transmission, Hyperalgesia, Anxiety

\section{Introduction}

Sustained abdominal pain is the most prominent feature of chronic pancreatitis (CP). It takes a heavy toll on the well-being of CP patients and renders a tough challenge for both gastroenterologists and pain physicians [1]. The management dilemma reflects a paucity of our knowledge concerning the pathogenesis of painful CP. The past decade has witnessed a conceptual transition from

\footnotetext{
* Correspondence: deptanat@fmmu.edu.cn; 277399335@qq.com

${ }^{\dagger}$ Yang Bai, Li-Tian Ma and Yan-Bing Chen contributed equally to this work. ${ }^{6}$ Department of Cardiology, The Second Affiliated Hospital of Xian Jiaotong University, Xian Jiaotong University, Xi'an 710004, China

'Department of Anatomy, Histology and Embryology \& K. K. Leung Brain Research Centre, Fourth Military Medical University, No. 169, West Chang-le Road, Xi'an 710032, China

Full list of author information is available at the end of the article
}

anatomical basis to neurobiological theory [2]. A growing body of evidence concerning the peripheral or spinal mechanisms of pancreatic pain identified a host of molecular targets, such as transient receptor potential vanilloid1 (TRPV1) and nerve growth factor (NGF), but corresponding therapeutic alternatives have not been applied into clinical practice owing to the lack of clinical evidence [3]. More importantly, owing to the highlyplastic nature of cortical synapses, enhanced peripheral inputs from the inflammatory pancreas trigger aberrant central pain processing, which perpetuates painful CP even after surgery or drugs aiming at eliminating peripheral barrage $[4,5]$. Considering these, we should embrace the ideology of cortical processing during painful $\mathrm{CP}$.

(c) The Author(s). 2019 Open Access This article is distributed under the terms of the Creative Commons Attribution 4.0 International License (http://creativecommons.org/licenses/by/4.0/), which permits unrestricted use, distribution, and 
Cerebral cortex is the ultimate hub for pain perception and sensitization, mediating the discriminative, affective and cognitive dimensions of pain [6]. Insights from electroencephalogram [7-9] and imaging studies [10, 11] provide evidence of structural and functional reorganizations of cerebral cortex, especially bilateral insular cortex (IC) and anterior cingulate cortex (ACC), in CP patients. IC is critical for the sensation, regulation and chronification of pain. Long-term potentiation (LTP) of insular excitatory synaptic transmission is believed to mediate pathological pain $[12,13]$. Glutamate is the major excitatory neurotransmitter within IC. Basic synaptic transmission is mainly mediated by $\alpha$-amino-3-hydroxy-5-methyl-4-isoxazole propionic acid receptor (AMPAR), while insular LTP requires both $N$-methyl-D-aspartate receptor (NMDAR) and AMPAR, which play important roles in the induction and expression phases, respectively [13]. Excitatory synaptic transmission within IC was enhanced during neuropathic pain, which was attributable to enhanced presynaptic release of glutamate and postsynaptic recruitment of AMPAR and NMDAR $[14,15]$. Thus, both insular lesion $[16,17]$ and drugs aiming at inhibiting insular glutamatergic transmission $[14,15,18,19]$ or plastic changes [20] exerted analgesic effects in neuropathic and inflammatory pain models. Recently, emerging evidence indicated the role of IC in stress-related visceral hypersensitivity [21-23]. However, whether there exist such neuroplastic changes within IC under the condition of painful $\mathrm{CP}$ receives few attentions.

Patients afflicted by CP usually exhibit pain-related comorbidities, such as emotional disorders and locomotor dysfunction [24, 25]. Unfortunately, much less focus has been directed on the relief of the affective dimension of pancreatitis pain in both clinical and preclinical researches. As a critical hub for both somatosensory and viscerosensory processing, IC is also important for emotional feelings, including emotional pain modulation [26, 27]. It is commonly recognized that anterior IC (aIC) is more involved in the emotional dimension of pain while posterior IC (pIC) participates in the sensory aspect of pain $[28,29]$. Considering these, we propose that aIC plays an important role in visceral hypersensitivity and pain-related negative emotions in painful $\mathrm{CP}$, which may provide important clues for clinical rehabilitative strategies for painful CP.

In the present work, we adopted abdominal withdraw threshold (AWT) and exploratory behavior in the open field as an indicator of anxiety, to evaluate the sensory and emotional aspects of painful $\mathrm{CP}$, respectively. Electrophysiological approaches were performed to explore CP-induced insular neuroplastic changes. Immunoblottings were applied to unravel the molecular basis for these plastic changes. Behavioral tests together with pharmacologic and chemogenetic methods aiming at manipulating the activity of aIC were performed to demonstrate its role in the pathogenesis of painful CP.

\section{Materials and methods \\ Animals and experimental design}

A total of 137 male Sprague-Dawley rats (250-280 g) were used, which were provided by the Experimental Animal Center of the Fourth Military Medical University (Xi'an, China). All protocols were performed according to the guidelines of the International Association for the Study of Pain [30] and approved by the Institutional Animal Care and Use Committee of the Fourth Military Medical University. Animals were provided water and food ad libitum $12 \mathrm{~h}$ before and after induction of pancreatitis, during which food and water were withdrawn.

Experiment 1: Evidence of pancreatitis-induced abdomen hyperalgesia: 27 rats were equally divided into 3 groups (naïve, sham and TNBS groups). Pancreatitis was induced by intraductal infusion of $2 \%$ trinitrobenzene sulfonic acid (TNBS; Sigma, St. Louis, MO, USA) as previously reported [31]. Sham rats received intraductal infusion of equal volume of saline. Naïve group received no surgery. AWT, as a measure of referred abdominal mechanical hypersensitivity, was an indirect marker of visceral sensitization [31]. In this experiment, AWT was monitored by von Frey filaments (VFF; Stoelting, Kiel, WI, USA) assay at post-operation day (POD) 3, 7, 14, 21, 28 and 35, respectively.

Experiment 2: Evidence of pancreatitis-induced hypolocomotion and effect of painful $\mathrm{CP}$ on the expressions of insular FOS, VGluT1, NR2B and GluR1. 53 rats were divided into 8 groups (6-7 rats in each group), which were exposed to intraductal TNBS or saline treatment. Open field test was performed to evaluate the locomotion and anxiety behavior according to our previous work [32] at POD 7, 14 and 28, respectively. After behavioral tests, rats were sacrificed. Heart blood was sampled and then centrifuged at $6000 \mathrm{~g}$ for $10 \mathrm{~min}$. Serum specimens were measured by amylase, lipase and total bilirubin activity assay kits (Sigma). Meanwhile, at least 3 rats in each group were processed for aIC sampling for VGluT1, NR2B and GluR1 immunoblotting. Other rats were perfused with $4 \%$ paraformaldehyde (PFA). Brain tissues were then sampled for FOS immunostaining and pancreatic tissues were sampled for $H \& E$ staining.

Experiment 3: Effect of painful CP on insular excitatory synaptic transmission. 16 rats were equally divided into 2 groups (sham and TNBS groups), which were exposed to intraductal saline or TNBS treatment, respectively. At POD 14, rats were sacrificed, and brain sections containing aIC were performed for electrophysiological recordings.

Experiment 4: Effect of CNQX and AP-5 microinjection into aIC on abdomen hypersensitivity of CP rats. 16 
rats were divided into 3 groups ( 5 in saline group, 5 in CNQX group and 6 in AP-5 group), all of which were exposed to intraductal TNBS treatment. For drug microinjection and behavioral test, the experimental paradigm was described in Fig. 8a.

Experiment 5: Effects of inactivation of aIC neural activity on abdomen hypersensitivity and anxiety of $\mathrm{CP}$ rats. To non-specifically modulate the activity of aIC neurons, 13 rats were divided into 2 groups, and received chemogenetic virus injection within aIC (7 rats in hSyn-mCitrine group and 6 in hSyn-Gi group). To further explore the role of aIC pyramidal neurons in painful $\mathrm{CP}, 12$ rats were equally divided into 2 groups (CaMKIIa-mCherry and CaMKIIa-Gi groups). All rats were exposed to intraductal TNBS treatment. For chemogenetic behavioral tests, the experimental designs were shown in Figs. 9a and 10a.

\section{Behavioral tests}

For VFF testing, the belly area designated for stimulation was shaved 3 days before testing. Animals were habituated in the testing apparatus until calming down. VFFs with increased forces from $0.16 \mathrm{~g}$ to $26 \mathrm{~g}$ were applied to the abdomen 5 times, each for $5-8 \mathrm{~s}$ with $5 \mathrm{~min}$ interval. Minimal force eliciting at least 3 times withdrawn responses was considered as AWT.

For open field test, rats were placed in the center of the open field $(100 \mathrm{~cm} \times 100 \mathrm{~cm} \times 60 \mathrm{~cm})$ under dim lighting. A motion-tracking system (Shanghai Mobile Datum Information Technology, CO. Ltd., Shanghai, China) was utilized to record the movement trace for 15 min. Anxiety-like behavior was evaluated by total traveling distance and the traveling distance in the center of the open field.

\section{Pancreatic H\&E staining}

For the verification of pancreatitis, pancreatic tissues were immersed in $4 \%$ PFA overnight, then $30 \%$ sucrose solution overnight, subsequently transferred to progressive xylene washes and embedded in paraffin. Paraffin blocks were cut at $8 \mu \mathrm{m}$ thickness and then stained with hematoxylin and eosin $(\mathrm{H} \& \mathrm{E})$ to evaluate pathological changes.

\section{Immunohistochemical staining}

On POD 14, TNBS and saline-treated rats were deeply anesthetized by i.p. administration of $7 \%$ chloral hydrate and then perfused with $50 \mathrm{ml}$ of $0.01 \mathrm{M}$ phosphate buffer saline (PBS, pH 7.4), followed by $500 \mathrm{ml} \mathrm{4 \%} \mathrm{PFA} \mathrm{fixative}$ solution in $0.1 \mathrm{M}$ phosphate buffer (PB, pH 7.4). After perfusion, brains were removed, placed in $30 \%$ sucrose solution for $24 \mathrm{~h}$ at $4{ }^{\circ} \mathrm{C}$ and then cut into coronal sections at $35 \mu \mathrm{m}$ thickness into 6 dishes as 6 sets of every sixth serial sections. The sections containing aIC in the first and second sets were performed immunohistochemical staining for FOS and double immunofluorescent staining for VGluT1 and NeuN, respectively. The sections were incubated in 10\% normal donkey serum (NDS) for $40 \mathrm{~min}$ at room temperature (RT) to block non-specific immunoreactivity, and then incubated overnight at $4{ }^{\circ} \mathrm{C}$ with following antibodies (shown in Table 1) in sequence: [1] primary antibodies for $18-24 \mathrm{~h}$ at $4{ }^{\circ} \mathrm{C}$; [2] secondary antibodies for $4 \mathrm{~h}$ at RT; or [3] avidinbiotin complex for $2 \mathrm{~h}$ (for FOS staining) at RT. For FOS staining, sections were then reacted with $0.05 \mathrm{M}$ Tris$\mathrm{HCl}$ buffer containing 0.02\% DAB (Dojin, Kumamoto, Japan) and $0.003 \% \mathrm{H}_{2} \mathrm{O}_{2}$ for visualizing FOS. Finally, the slices were mounted onto glass slides, coverslipped for examination by light microscopy (AH-3, Olympus, Tokyo, Japan) for FOS staining or confocal microscope (CLSM, FV1000, Olympus) for double immunostaining. The images were captured and analyzed using Fluoview 1000 (Olympus). For FOS counting, 15 sections from anterior (Bregma $+3.00 \sim+2.16$ ), middle (Bregma $+1.22 \sim$ +0.38 ) and posterior (Bregma $-1.14 \sim-1.98$ ) parts of IC (5 sections from each part) in one dish from each rat were counted for the total number of FOS-immunoreactive neurons in the three parts of IC between sham and TNBS groups ( $n=3$ rats in each group). For the determination of immunofluorescence intensity, nine microscopic images of aIC (with a $60 \mathrm{x}$ objective lens) were taken from three sections per animal. The mean intensity of VGluT1 immunofluorescence of the nine images per rat was measured in Image J ( $n=3$ rats in each group).

\section{Western blotting}

Insular tissues from four groups (sham, TNBS POD 7, 14 and 28 groups) were harvested in cold artificial cerebrospinal fluid (ACSF). Total protein (for VGluT1, NR2B and GluR1 assays) was prepared according to our previous study [33]. Membrane and cytoplasmic proteins (for NR2B, pNR2B, GluR1 and pGluR1 assays) were separated by Minute $^{\text {tix }}$ Plasma Membrane Protein Isolation Kit (Invent Biotechnologies, EdenPrairie, MN, USA) [34]. Subsequently, $30 \mu \mathrm{g}$ protein from each sample (quantitatively measured by bicinchoninic acid protein assay; Thermo Scientific; Rockford, IL, USA) was subjected to $10 \%$ sodium dodecyl sulfate-polyacrylamide gel (SDS-PAGE) electrophoresis and electrophoretically transferred to polyvinylidene difluoride (PVDF) membranes (Immobilon-P, Millipore, Billerica, MA, USA). After blocking in 5\% PBS-containing DifcoTM skim milk for $2 \mathrm{~h}$, the membranes were incubated overnight at $4{ }^{\circ} \mathrm{C}$ with following primary antibodies: mouse antiVGluT1 (1:500; Millipore); rabbit anti-pNR2B-Tyr ${ }^{1472}(1$ : 500; Millipore); rabbit anti-NR2B (1:500; Abcam, Cambridge, UK); rabbit anti-GluR1 (1:500; Millipore); rabbit anti-pGluR1-Ser ${ }^{845}$ (1:500; Millipore). The immunoblots were then incubated with corresponding horseradish 
Table 1 Antibodies used immunohistochemical staining

\begin{tabular}{llll}
\hline Antigens & Primary antibodies & Secondary antibodies & Tertiary antibodies \\
\hline FOS & Mouse anti-FOS & Biotinylated donkey anti-mouse & Avidin-biotin complex \\
& $(1: 500 ;$ Abcam, Cambridge, MA, USA) & $(1: 500 ;$ Millipore, Billerica, MA, USA) & (1:200; ABC kit, Vector, \\
& & Burlingame, CA, USA) \\
VGluT1/NeuN & Alexa594-donkey anti-mouse & \\
& Mouse anti-NeuN & $(1: 500 ;$ Invitrogen, Carlsbad, CA, USA) & \\
& (1:200; Millipore, Billerica, MA, USA) & Alexa488-donkey anti-rabbit & $(1: 500 ;$ Invitrogen, Carlsbad, CA, USA) \\
& Rabbit anti-VGluT1 & $(1: 300 ;$ Sysy, Gottingen, NI, GER) &
\end{tabular}

peroxidase (HRP)-conjugated secondary antibodies (goat anti-rabbit or goat anti-mouse, 1:5000; Amersham Pharmacia Biotech, Piscataway, NJ, USA). To verify equal loading, we also probed the membranes with rabbit anti-GAPDH (1:5000; Beijing TDY BIOTECH CO., Beijing, China) and rabbit anti-N-cadherin (1:2000; Millipore) for cytosol and membrane extracts, respectively. Bands were visualized by enhanced chemiluminescence (ECL) detection method (Amersham Pharmacia Biotech) and exposed to film. The scanned images were quantified and analyzed with Image J software. Target protein levels were normalized against GAPDH or N-cadherin levels and expressed as fold changes relative to those of the sham group $(n=3$ rats in each group).

\section{Extracellular field EPSP recordings}

For brain slice preparations, rats were anesthetized with $7 \%$ chloral hydrate and coronal brain sections $(300 \mu \mathrm{m})$ containing aIC were cut at $4{ }^{\circ} \mathrm{C}$ with a vibratome in oxygenated ACSF (in mM: $124 \mathrm{NaCl}, 2.5 \mathrm{KCl}, 2 \mathrm{MgSO}_{4}, 2$ $\mathrm{CaCl}_{2}, 25 \mathrm{NaHCO}_{3}, 1 \mathrm{NaH}_{2} \mathrm{PO}_{4}, 37$ glucose, $\mathrm{pH}$ 7.4). Then, the sections were transferred to a recovery chamber with oxygenated ACSF at RT for $1 \mathrm{~h}$ for subsequent electrophysiological experiments.

For extracellular field excitatory postsynaptic potential (fEPSP) recordings, a commercial 64-channel recording system (MED64, Panasonic, Osaka, Japan) was used. The MED64 probe (P515A, Panasonic) has an array of 64 planar microelectrodes arranged in an $8 \times 8$ pattern $(50 \times 50 \mu \mathrm{m}$ in size, interelectrode distance $150 \mu \mathrm{m})$, which could cover the insula (Fig. 3a, b). The preparation of MED64 probe was similar to our previous study [35]. The section was perfused with oxygenated ACSF at the rate of $2-3 \mathrm{ml} / \mathrm{min}$ with the aid of peristaltic pump (Minipuls ${ }^{\oplus}$, Inc., Gilson, France) during recording. After a 60-min recovery, one channel in the layers IV-V of aIC, from which the best synaptic responses could be elicited in surrounding recording channels, was selected as the stimulation site. Biphasic constant current pulses $(8-24 \mathrm{~mA}, 0.1 \mathrm{~ms})$ generated by the data acquisition software (Mobius, Panasonic Alpha-Med Sciences, Washington DC, USA) were applied to the stimulation site every minute. Testing intensity was determined when a half-maximal fEPSP was elicited in channels closest to the stimulation site. The fEPSPs evoked at both the superficial and deep layers were amplified by a 64-channel amplifier, displayed on the monitor screen, and stored on the hard disk of a microcomputer. After baseline synaptic responses were stabilized for $30 \mathrm{~min}$, a theta burst stimulation (TBS) protocol ( 5 bursts at $5 \mathrm{~Hz}$, repeated 5 times at $10 \mathrm{~s}$ intervals, 4 pulses at $100 \mathrm{~Hz}$ for each burst) was given at the test intensity to elicit post-synaptic LTP (post-LTP). After TBS, the test stimulus was repeatedly delivered every minute for $2 \mathrm{~h}$ to monitor the time course of LTP. For quantification of the LTP data, the fEPSPs measured by the slope (the rising phase between 10 and $90 \%$ of the peak response) and the amplitude were normalized as percent change from the baseline level. For comparison of LTP magnitude between two groups, the average value of the last $10 \mathrm{~min}$ recordings was compared statistically.

\section{Whole-cell patch-clamp recording}

For whole-cell patch-clamp recordings, the section was placed in a recording chamber maintained with oxygenated ACSF at $28^{\circ} \mathrm{C}$ and pyramidal neurons in the layers II-III of aIC were recorded. The potassium-based intracellular solution within the micropipettes (8-10 $\mathrm{M} \Omega$ ) contained the following (in $\mathrm{mM}$ ): $120 \mathrm{~K}$-gluconate, 5 $\mathrm{NaCl}$, 0.2 EGTA, 10 HEPES, 2 MgATP, $0.1 \mathrm{Na}_{3}$ GTP, 1 $\mathrm{MgCl}_{2}$ and 10 phosphocreatine (adjusted to $\mathrm{pH} 7.2$ with $\mathrm{KOH}, 290 \mathrm{mOsm})$. Spontaneous EPSCs (sEPSC) were recorded with membrane clamped at $-60 \mathrm{mV}$. To elicit synaptic responses in neurons within superficial layers, a bipolar tungsten stimulating electrode was placed within layer V (Fig. 4a). AMPA receptor-mediated EPSCs were elicited by repetitive stimulations every $10 \mathrm{~s}$ with neurons clamped at $-70 \mathrm{mV}$ in the presence of $50 \mu \mathrm{M}$ AP-5 (Tocris, Bristol, UK), while NMDA receptor-mediated responses were measured at $40 \mathrm{mV}$ in the presence of $20 \mu \mathrm{M}$ CNQX (Tocris). For paired-pulse facilitation (PPF) testing, the intervals were 35, 50, 75, 100 and 150 ms. To investigate the relationship between the spike number and the intensity of injected currents, pyramidal neurons were current-clamped and injected 50-350 pA depolarizing currents with a step-size of $50 \mathrm{pA}$. The rheobase current (minimum current to elicit an action 
potential) was explored via injecting depolarizing currents with a step-size of $2 \mathrm{pA}$.

For calculating the rectification of glutamate receptormediated EPSCs, cesium-based intracellular solution containing the following (in $\mathrm{mM}$ ): 122 Cs-Gluconate, 5 TEA-Cl, $3.7 \mathrm{NaCl}, 0.2$ EGTA, 20 HEPES, 0.3 MgATP, $0.3 \mathrm{Na}_{3} \mathrm{GTP}, 10$ BAPTA, 0.1 spermine and 5 QX-314 (adjusted to $\mathrm{pH} 7.2$ with $\mathrm{CsOH}, 290 \mathrm{mOsm}$ ) was used. The AMPA or NMDA receptor-mediated EPSCs were recorded at holding potentials of $50,30,10,0,-20,-40$ and $-60 \mathrm{mV}$. Then, the ratio of peak EPSC amplitudes at negative $(-40 \mathrm{mV})$ and positive $(50 \mathrm{mV})$ holding potentials was measured as rectification index.

All experiments were conducted in the presence of picrotoxin $\left(100 \mu \mathrm{M}\right.$, Sigma) to block $\mathrm{GABA}_{\mathrm{A}}$ receptormediated inhibitory synaptic currents. All recordings were performed with a MultiClamp 700B amplifier (Axon Instruments, Sunnyvale, CA, USA) and digitized at $5 \mathrm{kHz}$ by DigiData 1550B (Axon Instruments). The access resistance of $10-30 \mathrm{M} \Omega$ was monitored throughout the experiment. Data were discarded if the access resistance changed by $15 \%$ during experiment.

\section{Drug microinjection and behavioral tests}

7 days before TNBS treatment, rats were anesthetized and secured on a stereotaxic frame. Guide cannulas (26 gauge) were implanted bilaterally into aIC (AP: + 2.16, ML: \pm 5.0 , DV: $-6.2 \mathrm{~mm}$ ). On POD 14, intra-aIC injections were delivered through an injector cannula (30 gauge) which was located $0.2 \mathrm{~mm}$ lower than the guide. A Hamilton syringe $(10 \mu \mathrm{l})$ was connected to the injector by a thin polyethylene tube and was driven by a motorized pump (ALCBIO, Shanghai, China). AP-5 (50 mM, $0.4 \mu \mathrm{l}$ per side) or CNQX (20 mM, $0.4 \mu \mathrm{l}$ per side) was infused into bilateral $\mathrm{aIC}$ at the rate of $0.05 \mu \mathrm{l} / \mathrm{min}$, with an equivalent volume of saline as control. All injections were followed by an additional $5 \mathrm{~min}$ before removal of the injection cannula. AWT was measured before surgery, $30 \mathrm{~min}$ before and after drug microinjection at POD 14, as well as at POD 15. Injection sites were verified post hoc and rats with inaccurate sites were excluded.

\section{Chemogenetics and behavioral tests}

To non-specifically inhibit the activity of aIC neurons, we injected hSyn promoter-driven recombinant AAV virus (rAAV2/9-hSyn-hM4Di-mCitrine, $0.4 \mu \mathrm{l} / 30 \mathrm{~min}$, Taitool Bioscience, Shanghai, China) into bilateral aIC (AP: + 2.16, ML: \pm 5.0, DV: $-7.2 \mathrm{~mm}) 7$ days before TNBS treatment. An equivalent amount of rAAV2/9hSyn-mCitrine was injected into rats in sham group. To specifically inhibit the activity of aIC pyramidal neurons, we injected Calmodulin-dependent Protein Kinase IIa (CaMKIIa) promoter-driven virus (rAAV2/9-CaMKIIa-
hM4Di-mCherry, 0.4 $\mu \mathrm{l} / 30 \mathrm{~min}$, BrainVTA, Hubei, China) into bilateral aIC 7 days before TNBS treatment. An equivalent amount of rAAV2/9-CaMKIIa-mCherry was injected into rats in sham group. On POD 14, clozapine$\mathrm{N}$-oxide $(\mathrm{CNO}, 3 \mathrm{mg} / \mathrm{kg})$ was administrated intraperitoneally into the rats. AWT tests were performed before TNBS treatment, $1 \mathrm{~h}$ before and after $\mathrm{CNO}$ administration at POD 14, as well as at POD 15. Open field test was performed $2 \mathrm{~h}$ after $\mathrm{CNO}$ administration at POD 14. Injection sites were verified post hoc and rats with inaccurate sites were excluded.

\section{Data analysis}

Results were expressed as means \pm SEM. Statistical analyses between two groups were tested by Student's $t$-test (SPSS 17.0). Statistical comparisons between multiple groups were made using one-way ANOVA or one-way repeated ANOVA followed by LSD post-hoc test. Analyzed numbers for each experiment are indicated in corresponding figures $P<0.05$ was considered statistically significant.

\section{Results \\ TNBS-induced abdomen hypersensitivity and anxiety}

The validity of $\mathrm{CP}$ model was evidenced by histopathological changes of pancreatic tissue, such as acinar atrophy, inflammatory infiltration and stromal fibrosis (Fig. 1a-c). Owing to the damage of acinar cells, TNBS-treated rats exhibited increased contents of serum amylase and lipase on POD 3, which decreased along the course of $\mathrm{CP}$ and returned to baseline on POD 28 (Fig. 1d, e). In addition, TNBS-treated rats also exhibited high level of serum total bilirubin from POD 7 to 28 (Fig. 1f). All these changes mimicked those appeared in human chronic pancreatitis [36].

Then, behavioral assays were performed to provide evidence of abdomen hypersensitivity in CP rats. Sham group exhibited transient decrease of AWT which returned to baseline on POD 14, possibly owing to the abdominal incision during surgery. Comparing to sham group, TNBS-treated rats displayed a prolonged decrease in AWT from POD 7 to 35 (Fig. 1g). Open field testing showed that $\mathrm{CP}$ rats traveled less distance in the open field from POD 7 to 28, implicating the existence of hypolocomotion (Fig. 1h). Further analyses of open field results showed that $\mathrm{CP}$ rats traveled less distance in the central area along the course of $\mathrm{CP}$, suggesting the generation of anxiety-like emotions in $\mathrm{CP}$ rats (Fig. 1i). All these performance in the open field were consistent with those of mice with irritable bowel syndrome (IBS) observed in our previous study [32]. Considering these, apart from AWT as an indirect indicator of visceral hypersensitivity, we adopted hypolocomotion and decreased exploratory behavior to measure emotional pain modulation of $\mathrm{IC}$ in $\mathrm{CP}$ rats. 


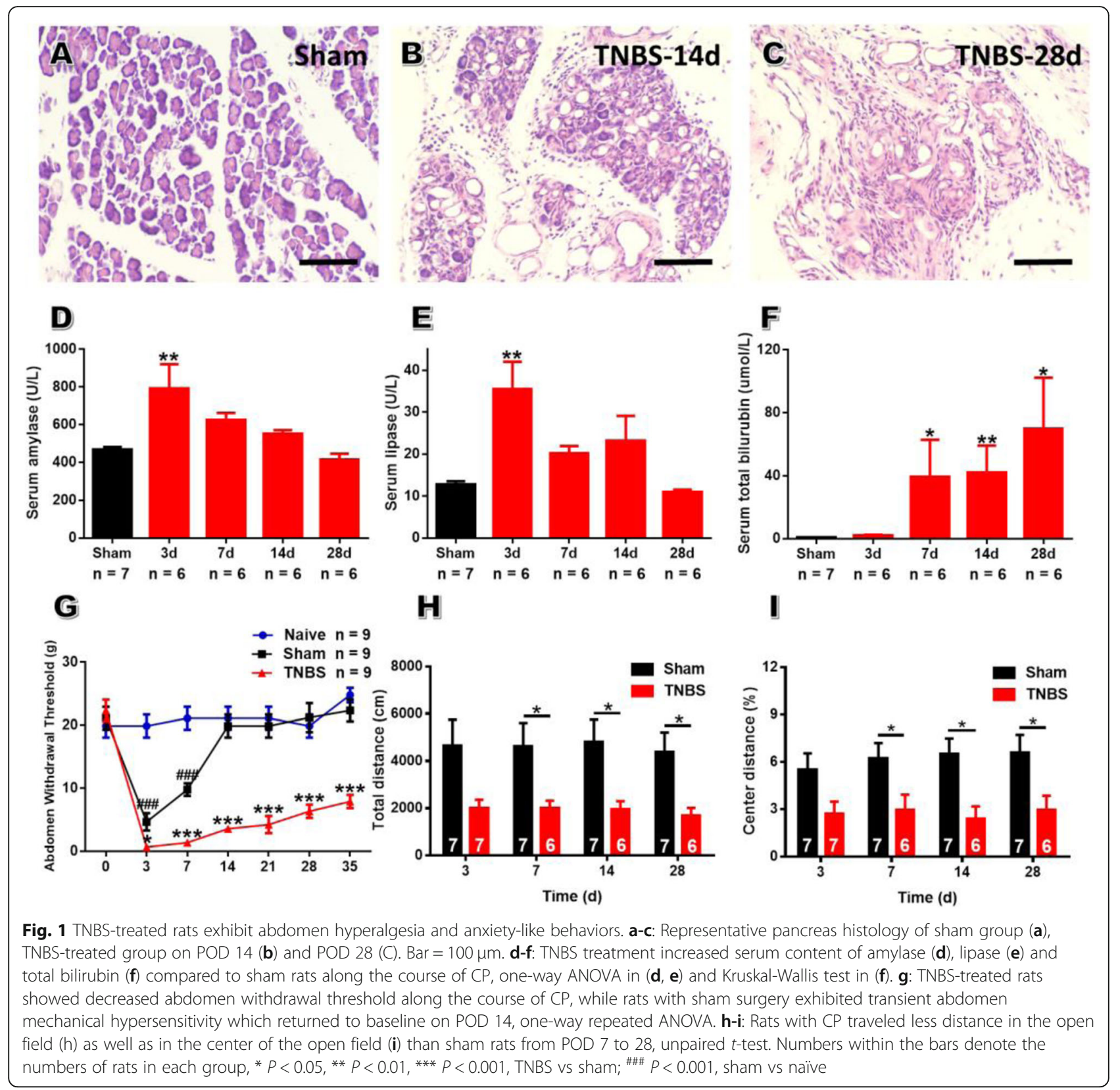

TNBS treatment increases FOS expression within IC

FOS is widely used as an indicator of neuronal activation under the condition of pain stimuli [37]. Our previous study indicated that IC exhibited increased number of FOS-expressing neurons in the IBS mouse model [32]. In the present study, FOS immunostaining was performed in brain sections containing IC at the time point of POD 14, when rats exhibited robust abdomen hyperalgesia and anxiety. As shown in Fig. 2a-c, TNBS treatment markedly increased insular FOS expression in both II-III and V-VI layers. Since $\mathrm{IC}$ is a long brain region along the rostro-caudal axis, we calculated the number of FOS-immunoreactive neurons in the anterior, middle and posterior parts of IC between sham and TNBS groups. Quantification data showed that TNBS treatment increased the number of FOS-expressing neurons within the whole IC, including anterior IC (TNBS: $2425 \pm 211.99$ vs saline: $679 \pm 65.39 ; P<0.01 ; n=3$ rats for each group), middle IC (TNBS: $2698.70 \pm 441.55$ vs saline: $806 \pm$ 161.36; $P<0.05$ ) and posterior IC (TNBS: $1517.30 \pm$ 246.81 vs saline: $393 \pm 60.02 ; P<0.05)$ (Fig. $2 d$ ). These data provided evidence for the activation of IC under the condition of painful $\mathrm{CP}$, laying morphological foundation for subsequent investigations of electrophysiological changes. 


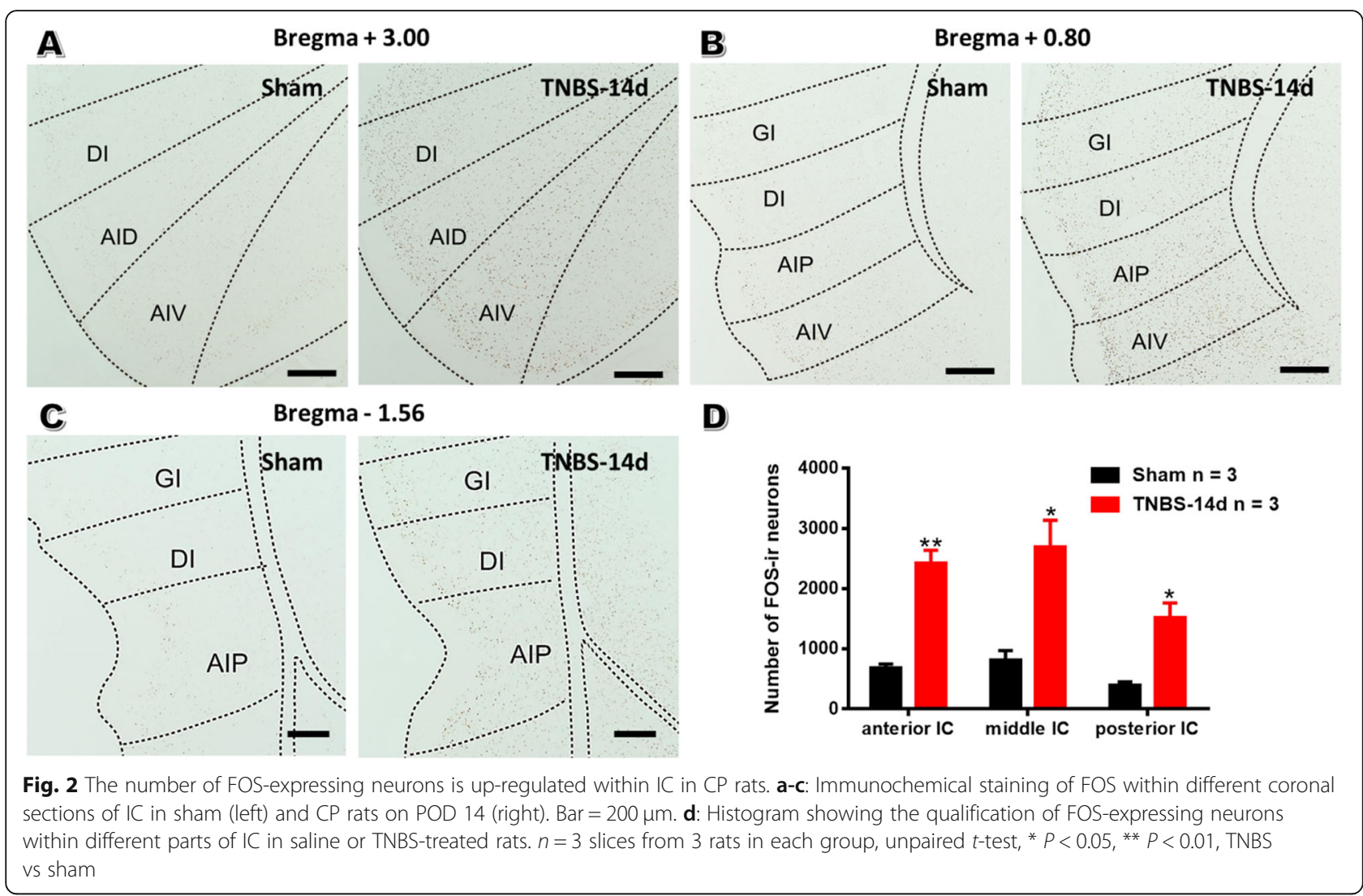

\section{Insular post-LTP is occluded after TNBS treatment}

The advantage of multi-electrode array recordings is to record multiple sites simultaneously, making it possible to examine insular neuroplastic changes in both space and time dimensions [35]. In subsequent studies, we concentrated on the anterior part of IC since it exhibited more obvious increase in FOS-expressing neurons in $\mathrm{CP}$ rats than other parts of IC. Moreover, aIC participates in both sensory and emotional aspects of chronic pain [28, 29]. We recorded sections containing aIC at the level of the corpus callosum connection ranging from bregma + $2.58 \sim+1.68 \mathrm{~mm}$ according to the rat atlas in stereotaxic coordinates [38]. The relative location of MED64 probe within the section was shown in Fig. 3a, b. Firstly, the input (stimulation intensity)-output (the number of activated channels) curve markedly shifted to the left in CP rats compared to sham group on POD 14 (Fig. 3c), indicating the hyperactivity of local insular neural network in CP rats. Secondly, as shown in Fig. 3d, we successfully induced post-LTP in both superficial and deeper layers within aIC after a TBS protocol applied in the deeper layer of aIC in sham rats. However, this potentiation was markedly reduced in the slices of $\mathrm{CP}$ rats (Fig. 3d-h, slope: $134.70 \pm 5.32 \%$ of baseline in CP rats vs $118.50 \pm 2.65 \%$ in sham rats; amplitude: $140.8 \pm 8.37 \%$ in $\mathrm{CP}$ rats vs $115.40 \pm 3.46 \%$ in sham rats; $P<0.05 ; n=6$ slices from 6 rats in each group). Finally, spatial analysis of post-LTP distribution indicated that CP rats displayed an obvious shrinkage of LTP map compared with sham group (Fig. 3i-j). This occlusive effect denotes that TNBS-triggered plasticity shares similar mechanisms with that of electrically-induced LTP.

\section{Enhanced neuronal activity within aIC of CP rats}

Then, we assessed electrophysiological changes of individual insular pyramidal neurons in layers II-III, which receive sensory inputs from cortical or subcortical areas, via whole-cell patch-clamp recordings on POD 14 after TNBS treatment (Fig. 4a). Pyramidal neurons were identified via spike frequency adaptation in response to prolonged depolarizing current injection (Fig. 4b). Firstly, we investigated the changes of neuronal activity under current-clamp mode. The input (the intensity of injection current)output (spike number) curve obviously shifted to the left in $\mathrm{CP}$ rats compared to sham rats (Fig. 4c, d). In addition, pyramidal neurons in $\mathrm{CP}$ rats showed reduced rheobase current and decreased resting membrane potential (Fig. 4e-g, Table 2). All these suggest the hyperexcitability of insular pyramidal neurons in $\mathrm{CP}$ rats. 
A

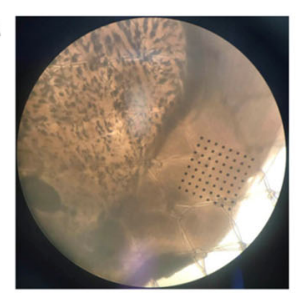

B

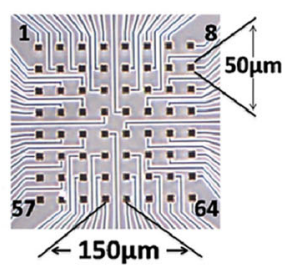

C

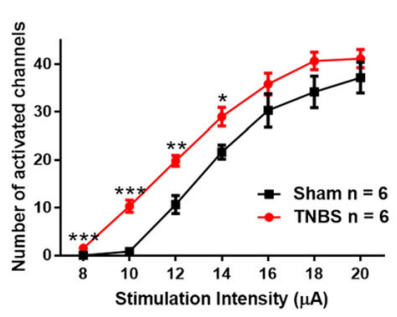

D

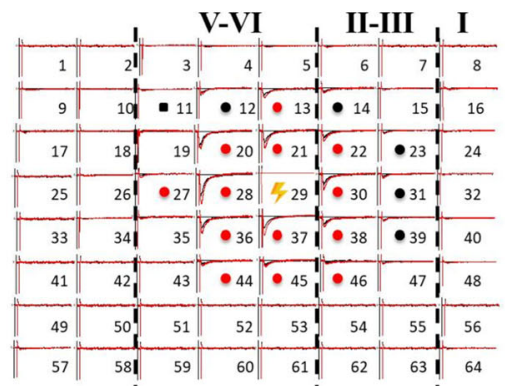

E

\begin{tabular}{|c|c|c|c|c|c|c|c|}
\hline & & \multicolumn{3}{|c|}{ V-VI } & \multicolumn{3}{|c|}{ II-III , I } \\
\hline 1 & 2 & 3 & 4 & $5 !$ & 6 & 7 & 8 \\
\hline 9 & 19 & $11 \|$ & 12 & 13) & 14 & 15 । & 16 \\
\hline $17 \mid$ & 18 & $19 \|$ & - 20 & -21 & $\cdot 22$ & 23 & 24 \\
\hline 25 & 26 & - 27 || & - 28 & - 29 & - 30 & $31 !$ & 32 \\
\hline 33 & $34 \mid$ & • 35 & - 36 & $|4,37|$ & $1 \cdot 38$ & 391 & 40 \\
\hline 41 & 42. & $43 \|$ & - 44 & $\bullet 45$ & $\cdot 46$ & 47 & 48 \\
\hline 49 & 50 & 51 & 52 & 53 & 54 & 55 & 56 \\
\hline $57 \mid$ & 581 & 59 & 60 & 61 & 162 & 631 & 64 \\
\hline
\end{tabular}

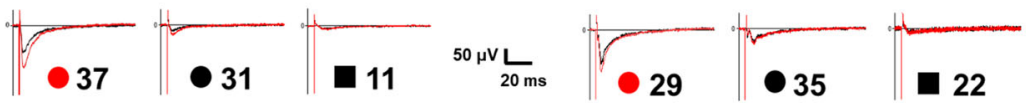

F

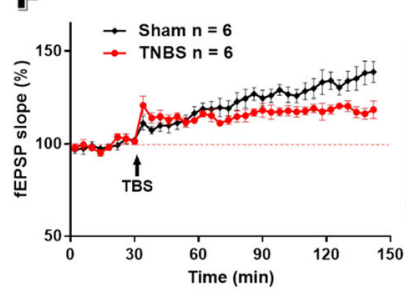

I

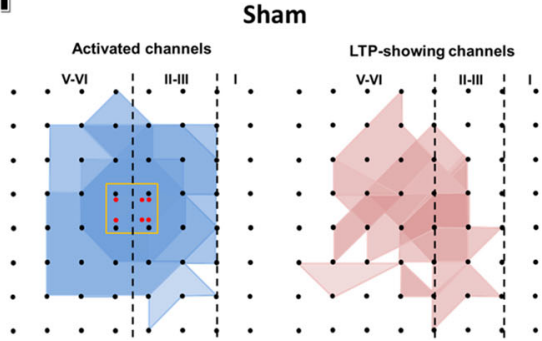

G

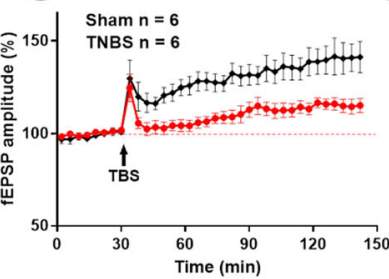

J
H

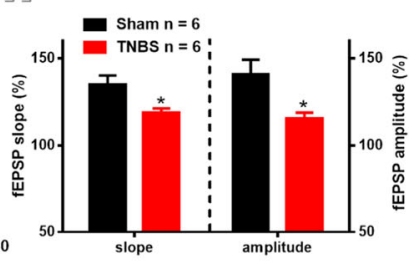

TNBS

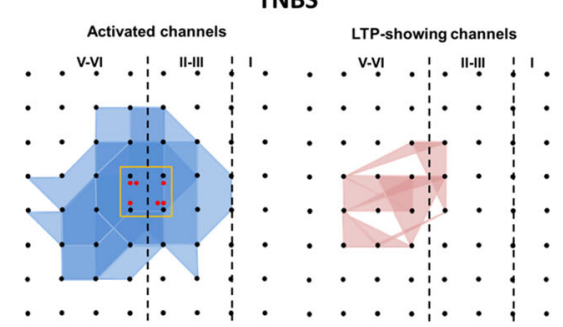

Fig. $\mathbf{3}$ Post-LTP is occluded within alC in rats with CP. a: Light microscopy photograph showing relative location of alC within the probe. $\mathbf{b}$ : Schematic diagram showing the recording array arrangement. $\mathbf{c}$ : The input-output curve of the number of activated channels in slices of sham and CP rats, one-way repeated ANOVA. d, e: A sample of an overview of multisite synaptic responses recorded at baseline (black) and $2 \mathrm{~h}$ after TBS (red) in sham and CP groups, respectively. The flash denotes the stimulated channel. Red and black filled circles mark all activated channels undergoing and not undergoing LTP, respectively, while the black rectangle represents a typical channel not exhibiting any response in the baseline. These example traces are shown in an enlarged scale below. Vertical lines demarcate different layers. $\mathbf{f}, \mathbf{g}$ : Time course of averaged fEPSP slope and amplitude of all active channels in sham and TNBS groups. The arrow indicates the time of TBS application. Dashed line indicates the baseline of 100\% slope or amplitude. $\mathbf{h}$ : The average slope and amplitude of fEPSPs of all active channels within the last 20 min of 140 min recording in sham and TNBS groups, unpaired t-test. $\mathbf{i}, \mathbf{j}$ : The polygonal diagram of activated (blue) and LTP-occurring (red) channels within alC after TBS in sham (i) and TNBS (j) groups. The red dots indicate the stimulation sites. $n=6$ slices from 6 rats in each group. ${ }^{*} P<0.05,{ }^{* *} P<0.01$, *** $P<0.001$, TNBS vs sham 


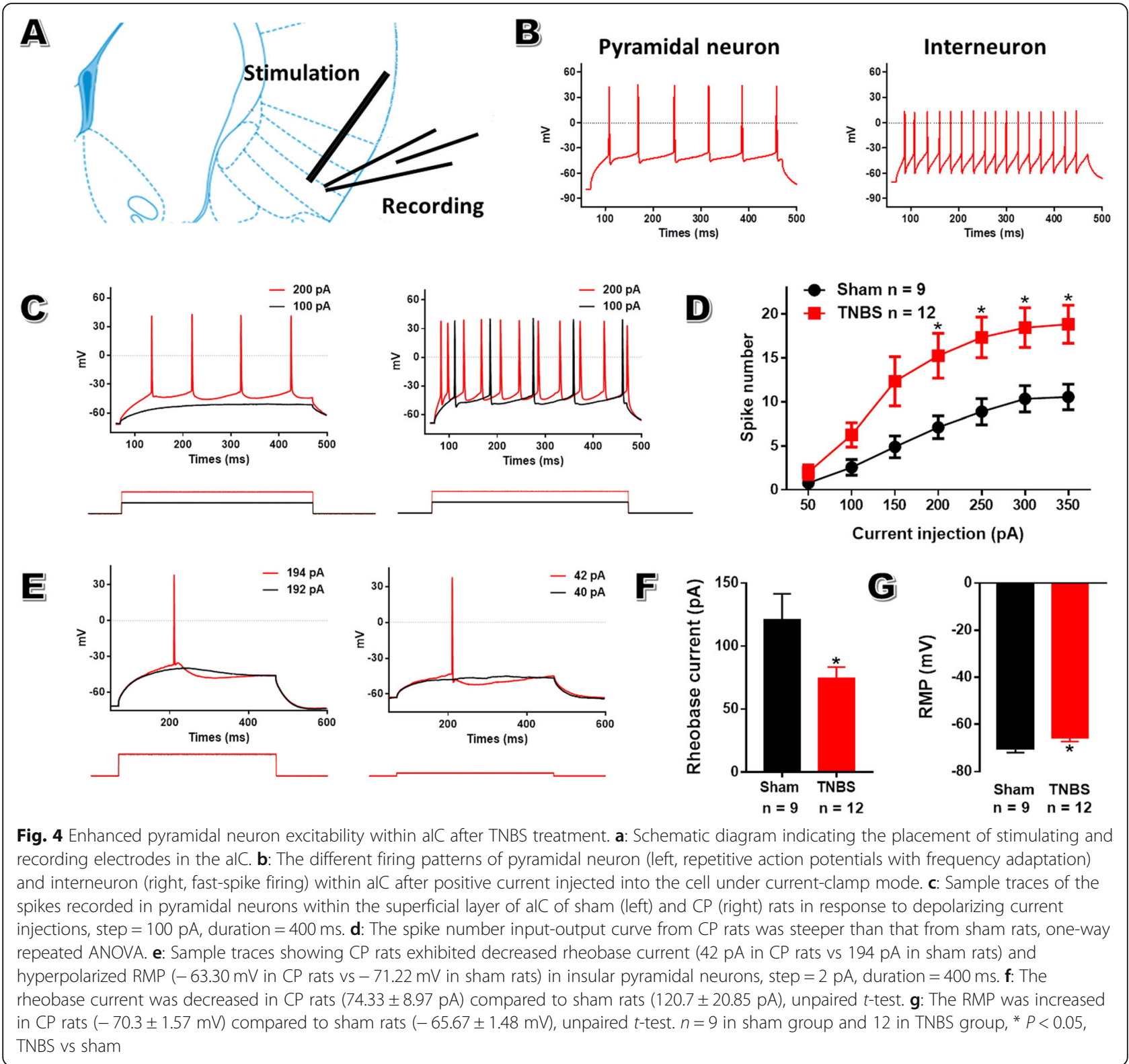

\section{Enhanced presynaptic transmitter release probability within aIC of CP rats}

To explore whether there exist any changes in basal synaptic transmission within aIC after TNBS treatment, sEPSCs were recorded under voltage-clamp mode. A robust augmentation in both frequency and amplitude was observed in CP rats (Fig. 5a-c, frequency: $1.46 \pm 0.28 \mathrm{~Hz}$ in $\mathrm{CP}$ rats vs $0.65 \pm 0.26 \mathrm{~Hz}$ in sham rats; amplitude: $18.48 \pm 0.84 \mathrm{pA}$ in $\mathrm{CP}$ rats vs $15.06 \pm 0.96 \mathrm{pA}$ in sham rats; $P<0.05 ; n=11$ cells in $C P$ group and 12 in sham group). These results suggest that enhanced presynaptic glutamate release and postsynaptic responsiveness both likely contribute to the enhanced excitatory synaptic transmission in the aIC of CP rats.
To verify that presynaptic mechanisms mediate the enhanced excitatory synaptic transmission in aIC, PPF, a measure of presynaptic function in which the response to the second stimulus is enhanced as a result of residual calcium in the presynaptic terminal after the first stimulus [39], was examined by electrical stimulation of the deeper layer of aIC. As seen in Fig. 5d, PPF was significantly reduced at time intervals of 35,50 and $75 \mathrm{~ms}$ in the aIC of CP rats compared to sham rats.

VGluT1 is necessary for excitatory synaptic transmission through glutamate packaging and exocytosis within cerebral cortex, the number of which has a major impact on quantal size in glutamatergic neurons [40]. To examine if there are expressional alterations of VGluT1 
Table 2 Summary of membrane properties of insular pyramidal neurons in sham and $\mathrm{CP}$ rats

\begin{tabular}{llll}
\hline & Sham group & CP group & $P$ \\
\hline Passive membrane properties & & & \\
Resting potential $(\mathrm{mV})$ & $-70.30 \pm 1.48$ & $-65.67 \pm 1.42$ & 0.048 \\
Membrane resistance $(\mathrm{M} \Omega)$ & $225.8 \pm 30.84$ & $201.33 \pm 22.35$ & n.s. \\
Access resistance $(\mathrm{M} \Omega)$ & $23.44 \pm 1.34$ & $23.13 \pm 2.00$ & n.s. \\
Membrane time constant (ms) & $2.07 \pm 0.26$ & $1.89 \pm 0.25$ & n.s. \\
Active membrane properties & & & \\
Current threshold (pA) & $120.67 \pm 19.65$ & $74.33 \pm 8.60$ & 0.038 \\
Voltage threshold (mV) & $-38.54 \pm 1.17$ & $-39.45 \pm 1.32$ & n.s. \\
AP amplitude (mV) & $82.91 \pm 2.31$ & $83.84 \pm 1.27$ & n.s. \\
AP rise slope (mV/ms) & $132.90 \pm 4.79$ & $123.22 \pm 5.15$ & n.s. \\
AP decay slope (mV/ms) & $-46.00 \pm 3.83$ & $-61.29 \pm 4.11$ & 0.021 \\
AP latency (ms) & $219.92 \pm 41.45$ & $384.42 \pm 85.92$ & n.s. \\
Cell capacitance (pF) & $82.81 \pm 9.72$ & $69.75 \pm 7.11$ & n.s. \\
\hline
\end{tabular}

Values are means \pm SEM. The level of significance was determined using Student's unpaired $t$ test. $P$ values for the comparison of insular pyramidal neurons in sham and $\mathrm{CP}$ rats

within aIC in $\mathrm{CP}$ rats, biochemical and morphological studies were performed. Biochemical results showed upregulated expression of VGluT1 along the course of CP (Fig. 5e), which was confirmed by immunohistochemical staining data showing more VGluT1-ir terminals were encountered in TNBS-treated rats on POD 14 than sham rats (Fig. 5f). These data suggest $\mathrm{CP}$ stimulates glutamate production and release, providing presynaptic evidence for enhanced synaptic transmission within aIC.

\section{Enhanced postsynaptic responsiveness of AMPAR and NMDAR within alC of CP rats}

To explore postsynaptic mechanisms underlying the enhanced excitatory synaptic transmission in the aIC of CP rats, we isolated AMPAR and NMDAR-mediated EPSCs in the presence of AP-5 and CNQX, respectively. Input (stimulation intensity) - output (EPSC amplitude) curves of both AMPAR (Fig. 6a) and NMDAR (Fig. 6b) -mediated currents were significantly shifted to the left after TNBS treatment, suggesting that both AMPAR and NMDAR-related mechanisms participate in the postsynaptic enhancement of aIC during painful $\mathrm{CP}$.

AMPARs channels are composed of four subunits. GluR2-containingAMPARs are impermeable to $\mathrm{Ca}^{2+}$ [41] while GluR2-lackingAMPARs possess high $\mathrm{Ca}^{2+}$ permeability and show inward-rectifying currents [42]. To determine whether TNBS treatment alters the prevalence of GluR2lacking and GluR2-containing AMPARs within aIC, we examined current-voltage relationship of AMPAR-EPSCs of insular pyramidal neurons. In sham rats, the AMPAREPSCs showed a near-linear $I-V$ relationship with a reversal potential near $0 \mathrm{mV}$, while the amplitude of AMPAR-
EPSCs of CP rats was reduced at positive membrane potentials (Fig. 6c). The rectification index $\left(\mathrm{I}_{-40 \mathrm{mV}} / \mathrm{I}_{+50 \mathrm{mV}}\right)$ of AMPAR-EPSCs was significantly increased in CP rats compared with sham rats $(1.37 \pm 0.31$ in CP rats vs $0.60 \pm 0.12$ in sham rats; $n=11$ neurons in CP rats and 10 in sham rats; $P<0.05$; Fig. $6 \mathrm{~d}$ ). All these data suggest chronic pancreatitis increases GluA2-lacking AMPAR prevalence in aIC pyramidal neurons. Moreover, the $I-V$ relationship of NMDARmediated EPSCs from sham group showed a typical outward rectification, which was similar to that of CP rats (Fig. 6e, f), suggesting no change in the characteristic of NMDAR-mediated currents during painful CP.

\section{Enhanced phosphorylation and membrane accumulation ofNR2B and GluR1 within alC in CP rats}

NMDAR channels are composed of two major subunits, including NR1 and NR2A-D. Among these, NR2B is the predominant subunits within $\mathrm{IC}$, which determines the trafficking and synaptic localization of NMDAR and plays an important role in chronic neuropathic pain [15, 43]. As the key subunit of AMPAR (including GluR1-4 subunits) within IC, GluR1 generates AMPAR trafficking and integration within synaptic membranes and contributes to the chronification of neuropathic pain [14, 44]. Then, we examined the expressional changes of these two representative glutamate receptor subunits, NR2B and GluR1, in CP rats. In consistent with our functional evidence, biochemical analysis showed that $\mathrm{CP}$ triggers long-term increase in the expression of AMPAR and NMDAR within aIC (Fig. 7a-c). All these data suggest both insular AMPAR and NMDAR are involved in postsynaptic enhancement during painful CP.

Both AMPAR and NMDAR are dynamically shifting in and out of membrane according to synaptic activity, which in turn determines synaptic strength $[45,46]$. To determine whether membrane glutamate receptors increased in $\mathrm{CP}$ rats, biochemical analyses were performed to measure the abundance of NR2B and GluR1 in both membrane and cytoplasmic fractions. A clear isolation of membrane and cytoplasmic protein was confirmed by the distribution of $\mathrm{N}$-Cadherin, a neural membrane marker, in membrane protein instead of its cytoplasmic counterpart (Fig. 7d). Biochemical analysis of NR2B within aIC at different time points showed membrane expression of NR2B was robustly up-regulated (Fig. 7e and g), while its cytoplasmic content was significantly down-regulated in $\mathrm{CP}$ rats as compared with sham group (Fig. $7 \mathrm{f}$ and $\mathrm{h}$ ). In addition, the abundance of membrane GluR1 was also markedly heightened in CP rats (Fig. 7e and i), with no striking change in that of cytoplasmic GluR1 (Fig. 7f and j). In line with increased inward rectification of AMPAR-mediated currents mentioned before, these data supports the increase of membrane calcium-permeable AMPARs in painful CP rats. 


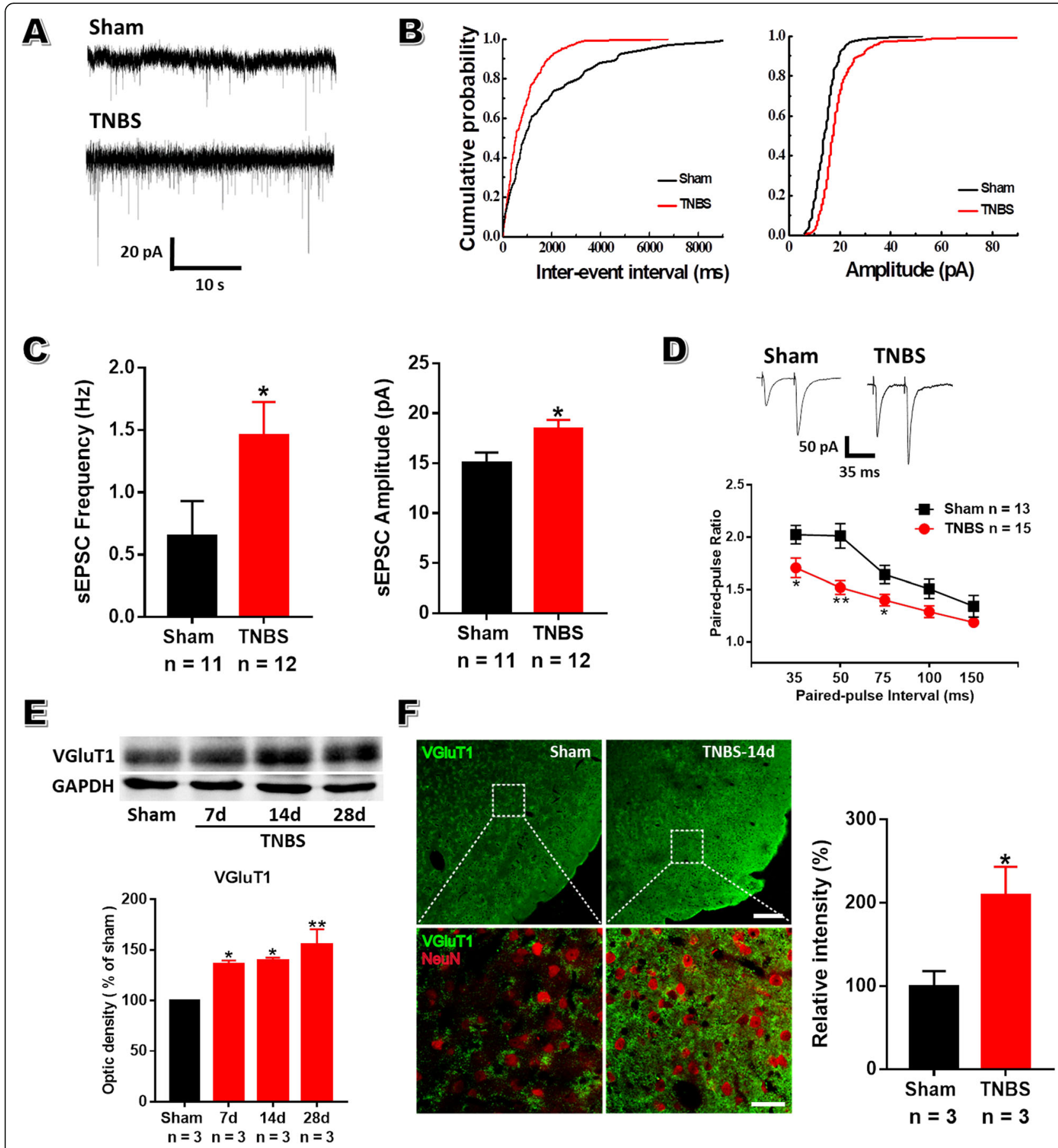

Fig. 5 Enhanced presynaptic glutamate release within alC after TNBS treatment. a: Representative sEPSCs recorded in the superficial layer of alC from sham (top) and TNBS-treated (bottom) rats holding at $-60 \mathrm{mV}$. b: Cumulative inter-event interval (left) and amplitude (right) histograms of sEPSCs recorded in the same neurons showed in (a). c: Summary plots showing the frequency (left) and the amplitude (right) of sEPSC was increased in CP rats $(1.46 \pm 0.28 \mathrm{~Hz}, 18.48 \pm 0.84 \mathrm{pA}, n=12)$ compared to sham rats $(0.65 \pm 0.26 \mathrm{~Hz}, 15.06 \pm 0.96 \mathrm{pA}, n=11)$, unpaired $t$-test. d: Representative traces of PPF with an interval of $35 \mathrm{~ms}$ recorded in the superficial layer of alC (top). PPF at time intervals of 35,50 and $75 \mathrm{~ms}$ was reduced in CP rats (bottom). $n=13$ cells in sham group and 15 in TNBS group, one-way repeated ANOVA. e: Representative western blot sample for VGluT1 within alC obtained on POD 7, 14 and 28 (top). Statistical analyses showing enhanced expression of VGluT1 from POD 7 to 28 after TNBS treatment (bottom). $n=3$ rats in each group, one-way ANOVA. f: The immunoreactivities of VGluT1 within alC were remarkably increased in TNBS-treated rats compared to sham rats. Microphotographs indicating double-immunoflurescence histochemistry for VGluT1 (green) and NeuN (red) within alC (left). The framed areas in upper images were magnified in lower images. Bars $=200 \mu \mathrm{m}$ in upper images and $40 \mu \mathrm{m}$ in lower images. Quantifications and statistical analyses of VGluT1 immunoreactivities presented in the graph (right). ${ }^{*} P<0.05,{ }^{* *} P<0.01$, TNBS vs sham 
A

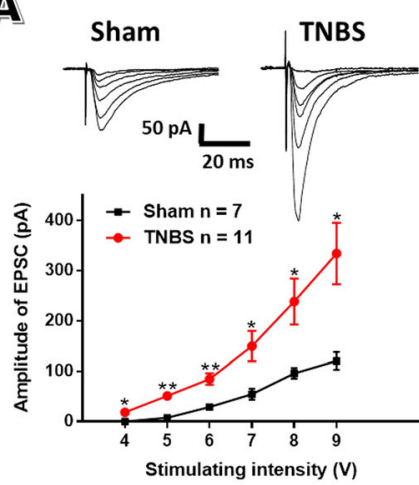

C
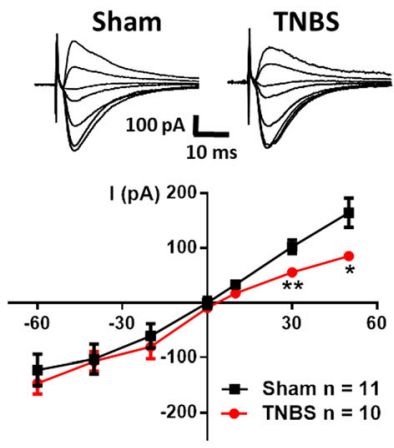

Holding potential ( $\mathrm{mV}$ )

E
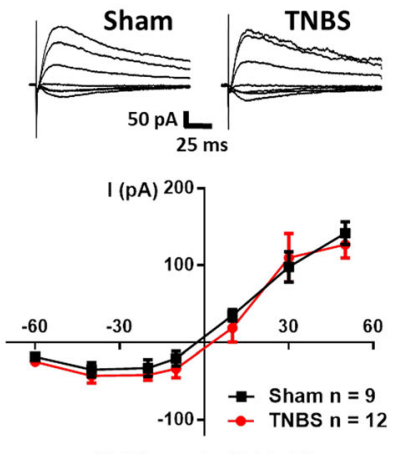

B

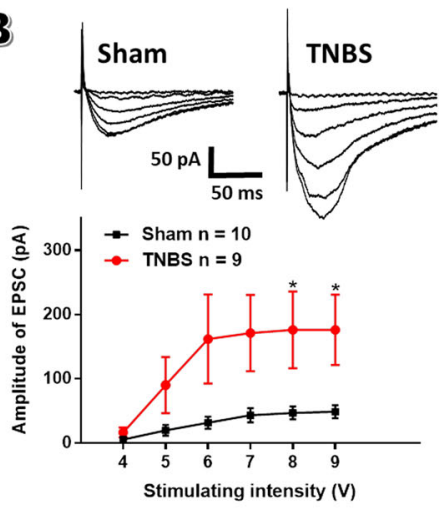

D

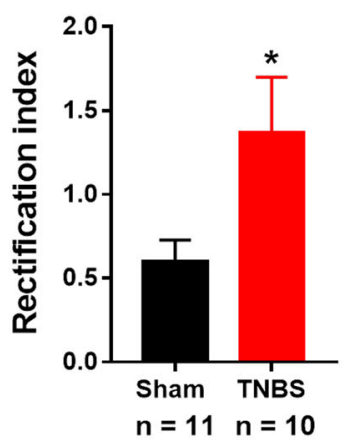

F

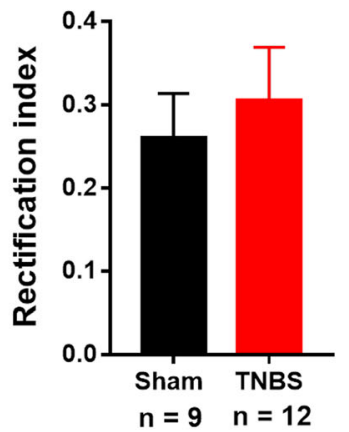

Fig. 6 Enhanced AMPAR and NMDAR currents within alC after TNBS treatment. a: The AMPAR-mediated synaptic input-output curve of CP rats $(n=11)$ was steeper than that from sham rats $(n=7)$, one-way repeated ANOVA. $\mathbf{b}$ : The NMDAR-mediated synaptic input-output curve of CP rats $(n=9)$ was steeper than that from sham rats $(n=10)$, one-way repeated ANOVA. c: I-V curves of AMPAR-EPSCs of alC pyramidal neurons recorded at holding potentials ranging from -60 to $+50 \mathrm{mV}$ in sham and CP rats, one-way repeated ANOVA. d: Comparison of the rectification index of AMPAR-EPSCs in sham $(n=11)$ and CP $(n=10)$ rats, unpaired $t$-test. e: $I-V$ curves of NMDAR-EPSCs of alC pyramidal neurons recorded at holding potentials ranging from -60 to $+50 \mathrm{mV}$ in sham and CP rats, one-way repeated ANOVA. $\mathbf{f}$ : Comparison of the rectification index of NMDAREPSCs in sham $(n=9)$ and CP $(n=12)$ rats, unpaired $t$-test. ${ }^{*} P<0.05,{ }^{* *} P<0.01$, TNBS vs sham

Phosphorylation is an important posttranslational modification for glutamateric receptors during their membrane targeting [43]. Phosphorylation of NMDAR, especially tyrosine phosphorylation of NR2B at $\mathrm{Tyr}^{1472}$ site, leads to enhanced synaptic NMPARs via inhibiting NMDAR endocytosis and mediates the chronification of neuropathic pain [15]. Likewise, serine phosphorylation of GluR1 at $\operatorname{Ser}^{845}$ site also contributes to the localization and function of insular AMPAR in mice with nerve injury [14]. To confirm whether similar posttranslational modifications occurred under the condition of painful $\mathrm{CP}$, we measured the phosphorylation status of membrane NR2B $\mathrm{Tyr}^{1472}$ site and GluR1 at $\operatorname{Ser}^{845}$ site using phosphorylation site specific antibodies. Membrane expression of $\mathrm{pNR} 2 \mathrm{~B}$ at $\mathrm{Tyr}^{1472}$ site was robustly ramped up along the course of CP (Fig. 7e and k), while no striking difference was detected in its cytoplasmic counterpart between sham 


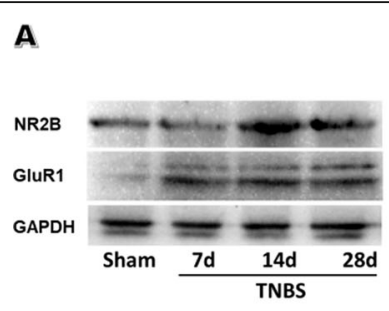

B

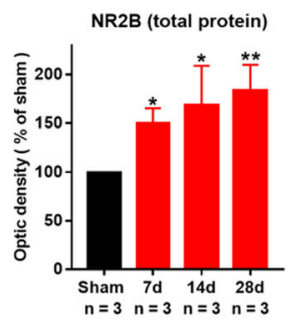

D

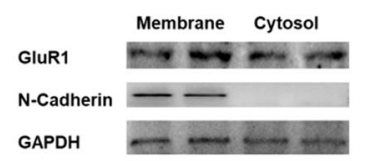

E

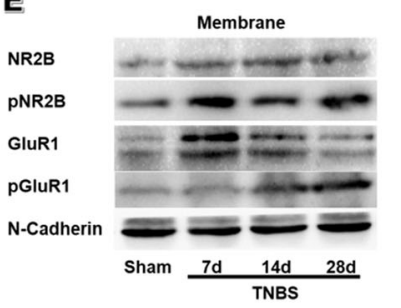

C

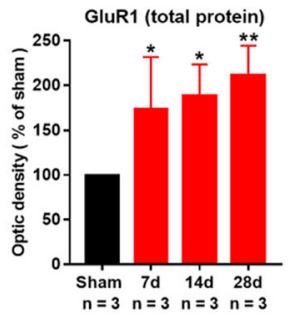

F

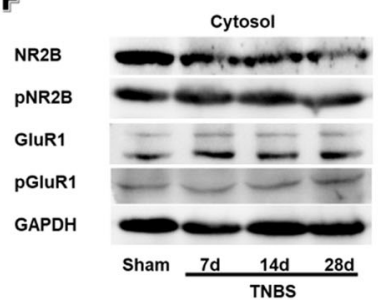

G

H

I
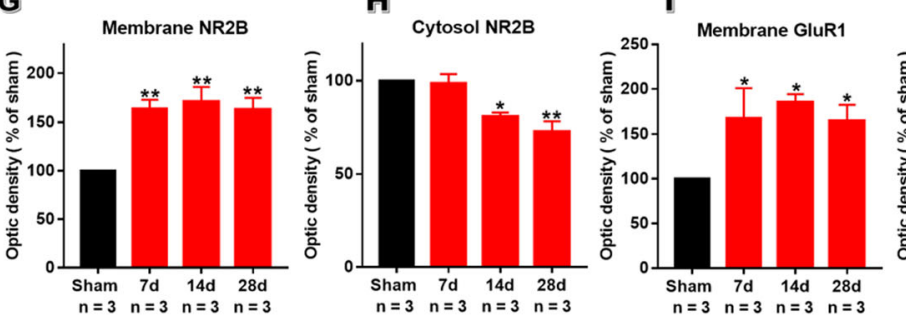

J

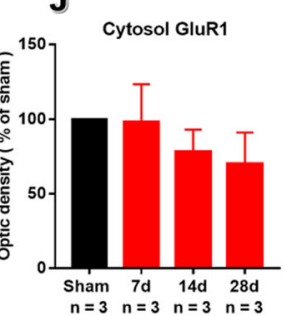

L
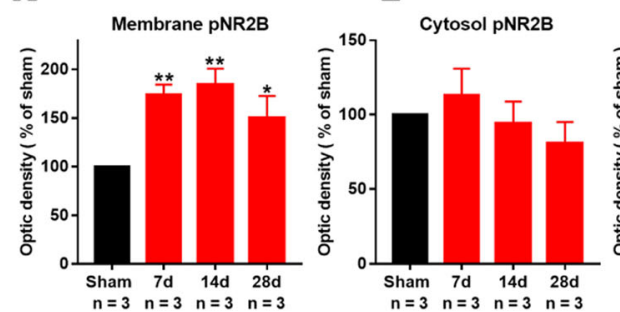

M

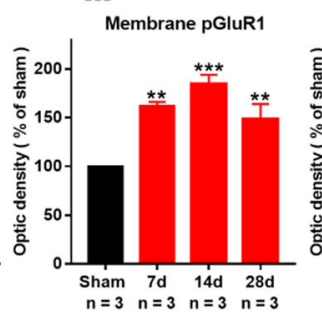

N

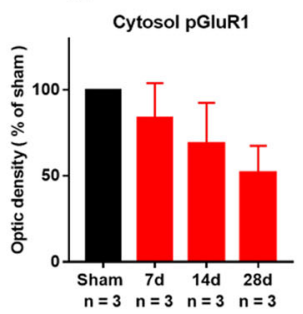

Fig. 7 TNBS treatment facilitates the phosphorylation and the trafficking of insular glutamate receptor subunits into membrane. a: Representative western blot for NR2B and GluR1 within alC on POD 7, 14 and 28. b, c: The expressions of NR2B (b) and GluR1 (c) were significantly enhanced from POD 7 to 28 after TNBS treatment. d: Fractionation of insular tissue was probed for N-cadherin and GAPDH to verify the accuracy of subcellular fractionation procedure. $\mathbf{e}$, $\mathbf{f}$ : Representative western blot samples for membrane (e) and cytosol (f) NR2B, pNR2B, GluR1 and pGluR1 within alC obtained on POD 7, 14 and 28 in TNBS-treated rats and sham rats. $\mathbf{g}, \mathbf{h}$ Membrane NR2B was significantly increased on POD 7, 14 and 28 after TNBS treatment compared to sham group while cytosol NR2B was significantly decreased on POD 14 and 28. $\mathbf{i}$, j Membrane GluR1 was significantly increased on POD 7, 14 and 28 after TNBS treatment compared to sham group while cytosol GluR1 showed no change. $\mathbf{k}$, Membrane pNR2B was significantly increased on POD 7, 14 and 28 after TNBS treatment compared to sham group while cytosol NR2B showed no change. $\mathbf{m}, \mathbf{n}$ Membrane pGluR1 was significantly increased on POD 7, 14 and 28 after TNBS treatment compared to sham group while cytosol pGluR1 showed no change. $n=3$ rats in each group, one-way ANOVA, ${ }^{*} P<0.05,{ }^{* *} P<0.01,{ }^{* *} P<0.001$, TNBS vs sham

and $\mathrm{CP}$ rats (Fig. $7 \mathrm{f}$ and $\mathrm{l}$ ). Furthermore, the content of membrane pGluR1 at $\operatorname{Ser}^{845}$ site also increased along the course of $\mathrm{CP}$ (Fig. 7e and $\mathrm{m}$ ), while cytoplasmic pGluR1 remained unchanged, albeit a tendency of declined expression of cytoplasmic pGluR1 in $\mathrm{CP}$ group (Fig. $7 \mathrm{f}$ and $\mathrm{n}$ ). All these demonstrated TNBS injection leads to the recruitment and modification of glutamate receptors, providing postsynaptic evidence for the enhanced synaptic transmission within aIC.

\section{Inhibiting excitatory synaptic transmission within alC} relieves abdomen hyperalgesia

To determine whether the enhanced excitatory transmission within aIC is detrimental in the development of pancreatitis pain, the effects of blocking insular glutamatergic transmission via CNQX and AP-5 on behavioral manifestations of pancreatic nociception were determined (Fig. 8a, b). Bilateral microinjection of CNQX and AP-5 into aIC reversed abdomen hyperalgesia in $\mathrm{CP}$ rats on POD 14 (AWT: $7.40 \pm 1.82 \mathrm{~g}$ for CNQX group, $5.67 \pm 0.87 \mathrm{~g}$ for 


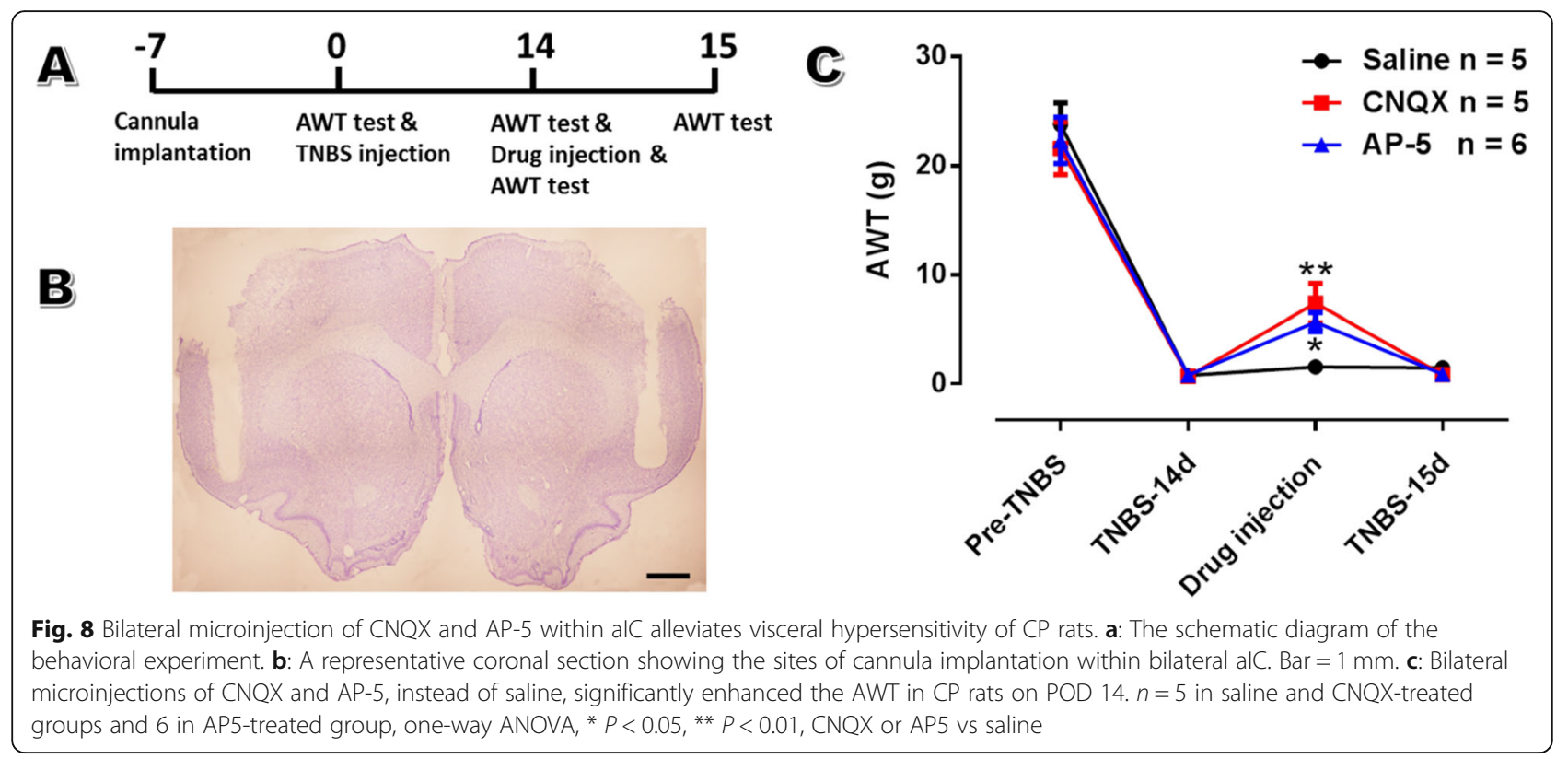

AP-5 group and $1.56 \pm 0.56 \mathrm{~g}$ for saline group; $n=6$ in AP- 5 group and 5 in both CNQX and saline groups; $P<$ 0.05 ; Fig. 8c), which returned to baseline on POD 15 $(0.84 \pm 0.26$ for CNQX group, $0.90 \pm 0.22$ for AP- 5 group and $1.48 \pm 0.30$ for saline group; $P>0.05$; Fig. $8 \mathrm{c}$ ). These behavioral results indicated the excitatory glutamatergic transmission within aIC contributes to hyperalgesia of $\mathrm{CP}$ rats.

\section{Inhibiting excitatory aIC neurons relieves abdomen hyperalgesia and anxiety}

Previous studies have substantiated that insular ablation could alleviate both neuropathic pain and stressrelated visceral pain $[16,17,23]$. In this study, we utilized chemogenetics to reversibly, non-specifically inhibit the activity of aIC neurons in CP rats. We targeted inhibitory designer receptor exclusively activated by designer drug (hM4Di DREADD) [47] to insular neurons by injecting recombinant adeno-associated vectors rAAV2/9-hSyn-hM4Di-mCitrine into aIC of $\mathrm{CP}$ rats (Fig. 9a, b). Behavioral results showed that non-selectively inhibiting insular neurons alleviated abdomen hyperalgesia of CP rats on POD 14 (AWT: $16.29 \pm 3.49 \mathrm{~g}$ in group and $3.86 \pm 1.10 \mathrm{~g}$ in mCitrine group; $n=6$ rats in Gi group and 7 in mCitrine group; $P<0.05$; Fig. 9c). No significant difference in baseline AWT was seen 3 days later after $\mathrm{CNO}$ treatment between these groups. To determine whether aIC is necessary for the resultant hypolocomotion and anxiety-like behavior, open field test was subsequently performed. Comparing to control group, bilateral insular inactivation did not influence animal locomotion in the open field, albeit an increasing tendency seen in $\mathrm{Gi}$ group (Fig. 9d). However, bilateral insular inactivation alleviated the decreased exploratory behavior of $\mathrm{CP}$ rats on POD 14 (Center distance \%: $3.63 \pm 1.06$ in Gi group and $0.28 \pm 0.11 \mathrm{~g}$ in mCitrine group; $P<0.01$; Fig. 9 e). All these suggest that aIC mediates hyperalgesia and pain-related anxiety in $\mathrm{CP}$ rats.

To verify whether the pain-facilitating role of aIC was mediated by excitatory pyramidal neurons, we used DREADDs to specifically suppress the activity of bilateral IC pyramidal neurons via CaMKIIa promoterdriven expression using rAAV vectors (rAAV2/9CaMKIIa-hM4Di-mCherry) under the condition of painful CP (Fig. 10a, b). Inactivation of aIC excitatory neurons elicited robust analgesic effects in CP rats on POD 14 (AWT: $11.33 \pm 2.77 \mathrm{~g}$ in Gi group and $3.67 \pm$ $0.56 \mathrm{~g}$ in mCitrine group; $n=6$ rats in each group; $P<0.05$; Fig. 10c). Interestingly, inhibiting the activity of aIC pyramidal neurons also alleviated the decrease in traveling distance as well as the exploratory behavior of $\mathrm{CP}$ rats in open field test (Fig. 10d, e). All these indicate an important role of insular pyramidal neurons in the production of hyperalgesia and anxiety during the process of $\mathrm{CP}$.

\section{Discussion}

In this study, we provided morphological, biochemical, physiological and behavioral evidence for that the excitatory synaptic transmission within aIC undergoes long-term potentiation and contributes to behavior sensitization during painful CP. Both presynaptic and postsynaptic mechanisms (the enhanced glutamate 


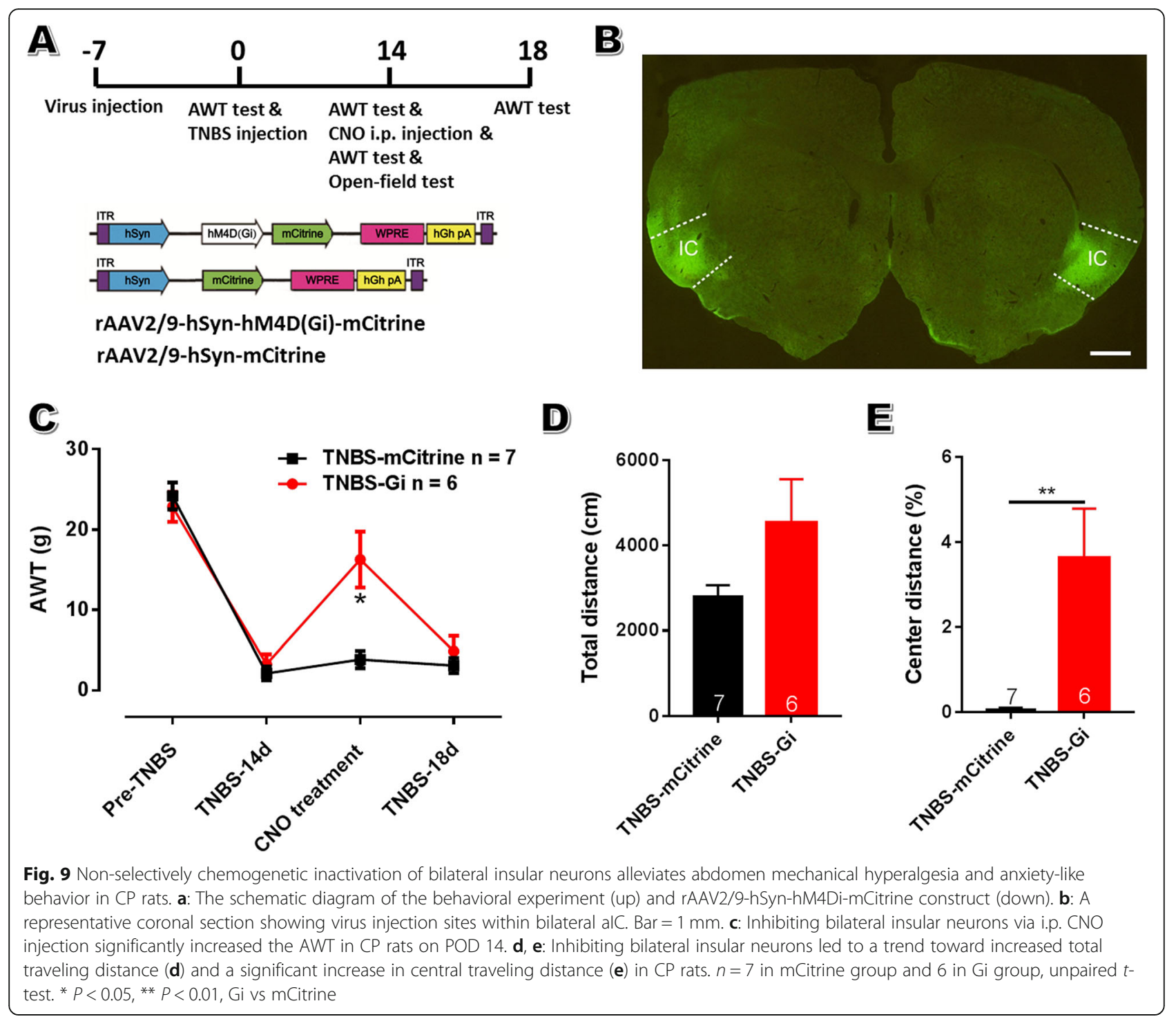

release and the recruitment of membrane glutamate receptors) are involved in this process. In addition, we further revealed that anterior insular pyramidal neurons exhibit hyperactivity under the condition of painful CP. Chemogenetic inhibition of aIC pyramidal neurons alleviates both hyperalgesia and pain-related anxiety in $\mathrm{CP}$ rats.

\section{Insular cortex and visceral pain}

Insular cortex plays an important role in the processing of somatic and visceral information, especially interoceptive signals from internal environment that reflects autonomic activity [26]. As a limbic integrating region, it is also considered critical for the generation of emotional feelings [27] and mood disorders, including anxiety and depression [48]. With regard to pain sensation, IC is a primary cerebral reception area for physiological visceral pain signals from the gut [49]. Accumulating imaging studies suggest IC also exhibits activity changes in chronic visceral pain states, such as IBS and bladder pain syndrome [50]. Under the condition of pancreatitis pain, what we know is that IC underwent both structural and functional reorganization $[7-9,11]$. However, the specific role of IC during painful CP is not clear. Recent preclinical researches indicated that, similar with the condition of neuropathic pain $[14,15]$, the excitatory transmission within aIC was enhanced in IBS rat model [21, 22] and bilateral aIC lesion could markedly inhibit the formation of visceral hypersensitivity [23]. Here, we observed that chronic pancreatitis triggered long-term up-regulation of glutamatergic 


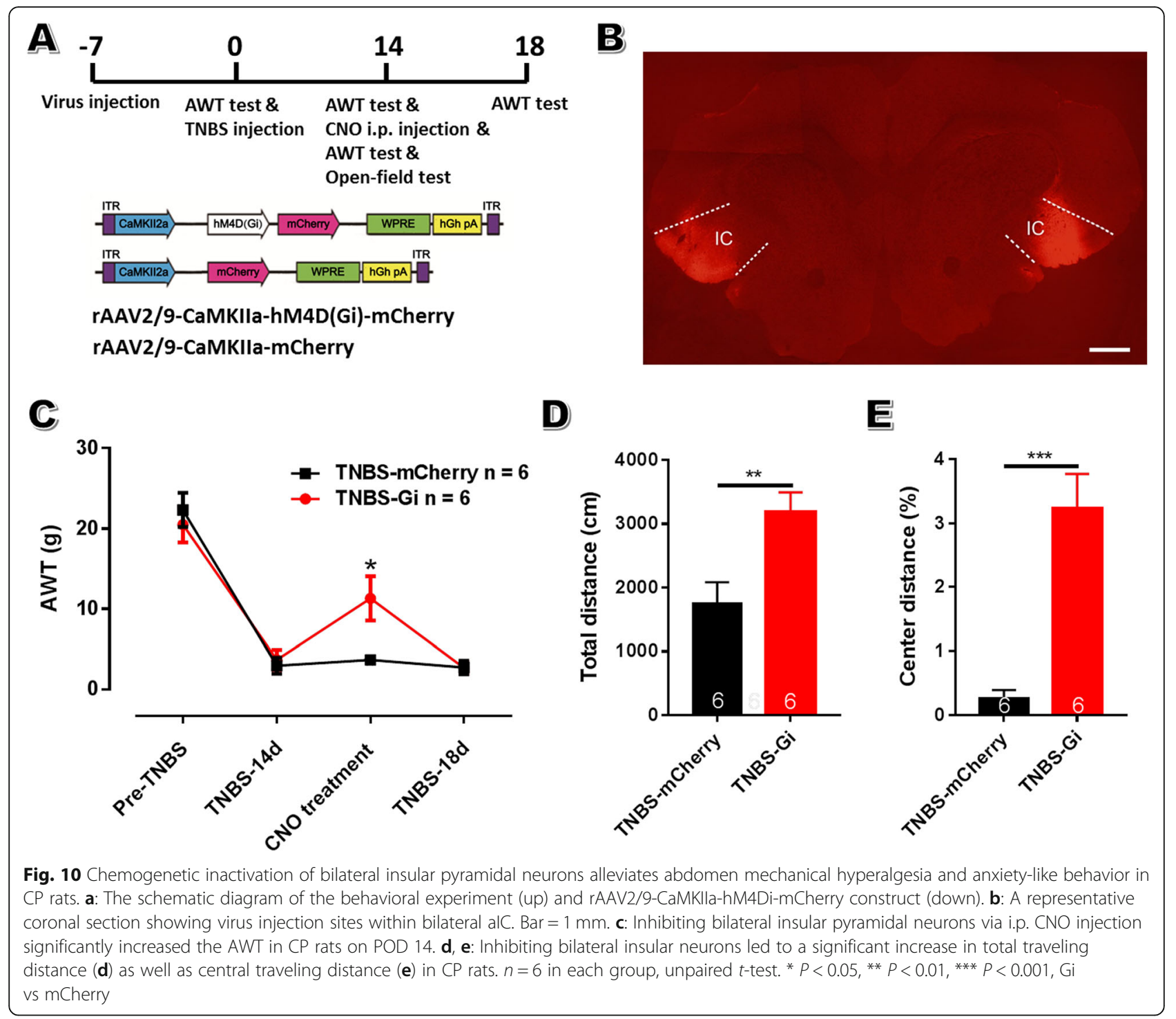

excitatory transmission and neural activity within the aIC, which may broaden our understanding of the role of IC in chronic visceral pain.

Chronic pain is frequently accompanied by affective disorders, which exacerbate the sensory abnormalities of chronic pain [51,52]. We observed long-term anxiety in TNBS-treated rats demonstrated by hypolocomotion and reduced exploratory behavior in the open field, which was also seen in IBS-related visceral pain [32]. This hypolocomotion is not owing to motor coordination disorder since rota rod test showed no difference in the falling latency between sham and $\mathrm{CP}$ rats (unpublished observations). It is worth noting that decreased locomotion is seldom seen in neuropathic pain [51]. One parsimonious explanation is that visceral pain is usually more unbearable than somatic pain and causes severer emotional and autonomic disorders, which may lead to decreased motor desire. Considering these, both hypolocomotion and exploratory behavior might be important indicators for affective disorders in painful CP. IC has been supposed to participate in emotional pain modulation. Based on the connectivity profile, it is considered that aIC preferably mediates its emotional effect while pIC is more involved in its somatosensory feature [12]. This theory was supported by the fact that pIC lesion reduced mechanical allodynia instead of the depressive consequences of chronic neuropathic pain [28]. Our study showed that the inactivation of aIC alleviated behavioral hypolocomotion and anxiety in $\mathrm{CP}$ rats, revealing the critical role of aIC in encoding the affective aspect of chronic pain. 


\section{Insular LTP and visceral pain}

Excitatory synapses are highly plastic, and LTP of glutamatergic transmission within IC is a key cellular mechanism for pathological pain [13]. Inducing LTPlike neuroplastic changes via injecting NMDA into IC in naïve rats produces mechanical allodynia [53], while inhibiting insular LTP via PKM inhibitor elicited analgesic effects under the condition of neuropathic pain [20]. In the present study, insular postLTP could be induced via TBS stimulation in sham rats instead of TNBS-treated rats. This failure of insular post-LTP induction was also encountered in mice with neuropathic pain [15]. All these suggest that, as similar with neuropathic pain, chronic pancreatitis pain may also recruit similar molecular mechanisms with TBS-induced LTP.

Activity-dependent $\mathrm{Ca}^{2+}$ flux through NMDAR activation, induced by excessive presynaptic glutamate release, is esteemed to initiate downstream signaling events during insular post-LTP induction [13, 54]. Adenylate cyclase 1 (AC1) is one essential $\mathrm{Ca}^{2+}$-stimulated enzyme that converts ATP to cAMP and activates downstream signaling molecules, such as protein kinase A (PKA) and cAMP-response element-binding protein (CREB). Evidence obtained from neuropathic pain indicates that nerve injury promotes insular GluR1 phosphorylation and trafficking. This phosphorylation of synaptic GluR1 is site-specific since only phosphorylation at $\operatorname{Ser}^{845}$ site (a PKA site) instead of $\mathrm{Ser}^{831}$ site (a CaMKII and PKC site) was increased, suggesting the pivotal role of AC1-cAMPPKA pathway under the condition of neuropathic pain [14]. Meanwhile, the activation of AC1-cAMPPKA pathway also facilitates insular NR2B phosphorylation specifically at $\mathrm{Tyr}^{1472}$ (not $\mathrm{Tyr}^{1336}$ or $\mathrm{Ser}^{1303}$ ) and forms a positive feedback to enhance NMDAR function, thus mediating neuropathic pain after nerve injury [15].

In the present study, our electrophysiological data provided functional evidence for presynaptic and postsynaptic amplifications within aIC in $\mathrm{CP}$ rats, which was verified by subsequent biochemical studies indicating enhanced expression of VGluT1 as well as membrane NR2B and calcium-permeable GluR1 subunits. In addition, the amounts of pGluR1 at $\mathrm{Ser}^{845}$ and pNR2B at $\mathrm{Tyr}^{1472}$ also increased within aIC. Considering these, we proposed that excessive glutamate release induced by pancreatitis pain may trigger calcium influx into post-synaptic membrane and subsequent intracellular signaling pathways to phosphorylate GluA1containing AMPA receptor and NR2B-containing NMDA receptor, which promotes membrane trafficking and long-term potentiation of insular excitatory transmission. During this process, AC1-cAMP-PKA pathway may play an important role in the modification and recruitment of glutamate receptors. These LTP-like neuroplastic changes are closely related to behavior allodynia since inhibiting insular plasticity via NMDAR and AMPAR antagonists relieved neuropathic pain $[14,15]$ as well as CP-related hyperalgesia in this study. These results provide novel cortical mechanisms for chronic visceral pain and shed light on drugs targeting at insular post-LTP, for example, the $\mathrm{AC1}$ inhibitor NB001 [32] in the treatment of painful CP. One limitation of our study is that we did not examine whether other glutamate receptor subunits and phosphorylation sites are involved in painful CP. Detailed signaling mechanisms also warrant our further investigation.

Another limitation of this study is that we only observed the occlusive effects of insular post-LTP under the condition of painful CP. Apart from post-LTP, presynaptic LTP (pre-LTP) is a kind of NMDARindependent LTP initially reported in hippocampus [55]. In 2015, this kind of LTP was detected within ACC by Koga et al. They further observed that this kind of LTP was occluded under the condition of chronic pain and anxiety, and erasing ACC pre-LTP exerted anxiolytic effects during chronic pain [56]. In light of these, pre-LTP is thought to mediates anxiety signals triggered by chronic pain, while post-LTP is related to behavioral hyperalgesia during chronic pain [57]. In a recent study, Zhuo $M$ et al. succeeded in recording pre-LTP in the insular cortex, which shared similar molecular mechanisms with ACC pre-LTP [58]. In the present study, we observed an increase in presynaptic glutamate release within aIC, suggesting the possibility of the existence of insular pre-LTP during painful $\mathrm{CP}$. Considering that both ACC and aIC are involved in emotional pain regulation, we propose that insular pre-LTP may also be involved in pain-related anxiety, which merits investigations in our future study.

\section{Neuromodulation techniques targeting IC as a viable therapy for painful CP}

Generally speaking, glutamatergic pyramidal neurons and GABAergic interneurons are two major groups within IC [6]. Pyramidal neurons within superficial layers receive emotional nociceptive and visceral inputs from medial thalamus while those within deep layers project toward subcortical structures for descending pain control. Within local neurocircuitry, interneurons release GABA to inhibit insular output activity via feedforward inhibition $[6,12]$. Jasmin $\mathrm{L}$ et al. observed that selectively activating insular pyramidal neurons within layer $\mathrm{V}$ via $\mathrm{GABA}_{\mathrm{B}}$ receptor antagonist elicits hyperalgesia while inhibiting insular output activity via increasing local GABA content produces 
lasting analgesic effects in naïve rats [18]. In the present study, our functional evidence showed that TNBS treatment robustly elevated the activity of aIC pyramidal cells and chemogenetic inactivation of pyramidal neurons alleviated both hyperalgesia and reduced exploratory behavior in $\mathrm{CP}$ rats. Considering these, we concluded that aIC glutamateric neurons mediate hyperalgesia as well as painrelated anxiety under the condition of painful $\mathrm{CP}$.

Another limitation of our study is that we did not examine whether activating insular neurons could elicit visceral pain in naïve rats owing to the limitation of our pain evaluating method. Since VFF probing is a measure of referred abdominal mechanical hypersensitivity when pancreatic inflammation invades the peritoneum [59], it may not be an efficacious index of internal visceral sensation under normal state. Thus, observing pancreas stimulation induced defensive behaviors via intra-abdominal electrodes [31] may be a better choice in our future studies to evaluate the sensory aspect of pancreatitis pain.

Historically, surgeries aiming at reducing ductal hypertension were introduced to treat painful $\mathrm{CP}$ in clinic. Nevertheless, it is not recommended in this day and age since ductal decompression failed to inhibit already existed central sensitization [60]. The cornerstone in the treatment of painful CP relies on multifunctional drugs, including opiates, antidepressants and antiepileptics, which provides favorable outcomes yet inevitably unbearable adverse effects, such as cognitive impairment and drug addiction [5]. Fortunately, the advent of brain stimulation, especially non-invasive paradigms including repetitive transcranial magnetic stimulation (rTMS) and transcranial direct current stimulation (tDCS), produces a promising alternative for modifying dysfunctional painmatrix activity in the treatment of refractory pain disorders [61]. Recent technical improvements in the development of cooled double-cone coils in rTMS opened the possibility to stimulate deeper cortical regions, such as insula $[62,63]$. Among various brain targets, such as prefrontal cortex and primary motor cortex $[64,65], \mathrm{IC}$ is a newly-identified spot for the intervention of chronic pain. It is well-documented that insular stimulation relieves both experimentallyinduced pain $[62,66,67]$ and neuropathic pain $[68]$. The preponderant theory for this is high-frequency stimulation generated neural depolarization blockade [67, 69] or the recruitment of local GABAergic circuit [66], leading to functional inactivation of insular neurons. In the present study, we successfully relieved pancreatitis pain and pain-related anxiety in $\mathrm{CP}$ rats through chemogenetic inhibition of aIC neuronal activity. These data may lay preclinical basis for the application of insular stimulation in the remedy of pharmacoresistant pancreatitis pain, which may outperform motor cortex stimulation via targeting the affective component of pain.
In conclusion, the data provided in the present work pinpoint the role of cortical sensitization and modulation in pancreatic visceral pain, supporting insula, especially its anterior region (aIC), as a potential candidate alleviating visceral hyperalgesia and related affective disorders. Given the prosperity of the domain of neuromodulation this era has witnessed, insular stimulation may yield tremendous benefits for those suffering from visceral pain and should be considered by gastroenterologists in the treatment of CP.

\section{Abbreviations}

ACC: Anterior cingulate cortex; alC: Anterior insular cortex; AMPAR: a-amino3-hydroxy-5-methyl-4-isoxazole propionic acid receptor; AWT: Abdominal withdraw threshold; CP: Chronic pancreatitis; CREB: CAMP-response elementbinding protein; fEPSP: Field excitatory postsynaptic potential; IBS: Irritable bowel syndrome; IC: Insular cortex; LTP: Long-term potentiation; NMDAR: Nmethyl-D-aspartate receptor; pIC: Posterior insular cortex; PKA: Protein kinase A; POD: Post-operation day; PPF: Paired-pulse facilitation; sEPSC: Spontaneous EPSCS; TBS: Theta burst stimulation; TNBS: Trinitrobenzene sulfonic acid; VFF: von Frey filaments

\section{Acknowledgements}

We thank Haipeng Su for his support on the measurement of serum amylase, lipase and total bilirubin.

\section{Authors' contributions \\ YB and YC established the CP model, performed behavior tests and harvested tissue samples. LM, YL and HS carried out immunoblotting tests. $B F$ and $Y C$ performed electrophysiological tests. DR carried out pancreatic H\&E staining. YB and TZ performed immunostaining tests. MZ and TC gave advice on electrophysiological data analysis. YL, XY and HL designed study and edited manuscript. YB analyzed data and wrote the manuscript. All authors read and approved the final manuscript.}

\section{Funding}

This work was supported by National Natural Science Foundations of China (grant number 81620108008) and Hainan ZDYF2018153.

\section{Availability of data and materials}

The datasets supporting the conclusion of this study are included in this article.

\section{Ethics approval}

All experiments are approved by the Institutional Animal Care and Use Committee of the Fourth Military Medical University.

\section{Consent for publication}

All of the authors have given their consent for publication.

\section{Competing interests}

The authors declare that they have no competing interests.

\section{Author details}

${ }^{1}$ Department of Anatomy, Histology and Embryology \& K. K. Leung Brain Research Centre, Fourth Military Medical University, No. 169, West Chang-le Road, Xi'an 710032, China. ${ }^{2}$ Department of Gastroenterology, Tangdu Hospital, Fourth Military Medical University, Xi'an 710032, China. ${ }^{3}$ Department of Anatomy, Fujian Medical University, Fuzhou 350108, China. ${ }^{4}$ Department of Anatomy, Guangxi Medical University, Nanning 530021, China.

${ }^{5}$ Department of Anatomy, Fujian Health College, Fuzhou 350101, China. ${ }^{6}$ Department of Cardiology, The Second Affiliated Hospital of Xian Jiaotong

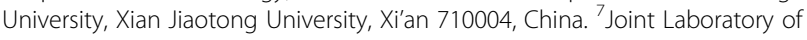
Neuroscience at Hainan Medical University and Fourth Military Medical University, Hainan Medical University, Haikou 571199, China. 


\section{Received: 30 June 2019 Accepted: 23 August 2019}

\section{Published online: 04 September 2019}

\section{References}

1. Olesen SS, Juel J, Nielsen AK, Frokjaer JB, Wilder-Smith OH, Drewes AM. Pain severity reduces life quality in chronic pancreatitis: implications for design of future outcome trials. Pancreatology. 2014;14(6):497-502.

2. Atsawarungruangkit $A$, Pongprasobchai $S$. Current understanding of the neuropathophysiology of pain in chronic pancreatitis. World J Gastrointest Pathophysiol. 2015;6(4):193-202.

3. Pasricha PJ. Unraveling the mystery of pain in chronic pancreatitis. Nat Rev Gastroenterol Hepatol. 2012;9(3):140-51.

4. Fregni F, Pascual-Leone A, Freedman SD. Pain in chronic pancreatitis: a salutogenic mechanism or a maladaptive brain response? Pancreatology. 2007;7(5-6):411-22.

5. Drewes AM, Krarup AL, Detlefsen S, Malmstrom ML, Dimcevski G, FunchJensen $P$. Pain in chronic pancreatitis: the role of neuropathic pain mechanisms. Gut. 2008:57(11):1616-27.

6. Zhuo M. Cortical excitation and chronic pain. Trends Neurosci. 2008;31(4): 199-207.

7. Dimcevski G, Sami SA, Funch-Jensen P, Le Pera D, Valeriani M, ArendtNielsen $\mathrm{L}$, et al. Pain in chronic pancreatitis: the role of reorganization in the central nervous system. Gastroenterology. 2007;132(4):1546-56.

8. de Vries $\mathrm{M}$, Wilder-Smith $\mathrm{OH}$, Jongsma ML, van den Broeke EN, Arns $\mathrm{M}$, van Goor $\mathrm{H}$, et al. Altered resting state EEG in chronic pancreatitis patients: toward a marker for chronic pain. J Pain Res. 2013;6:815-24.

9. Olesen SS, Frokjaer JB, Lelic D, Valeriani M, Drewes AM. Pain-associated adaptive cortical reorganisation in chronic pancreatitis. Pancreatology. 2010; 10(6):742-51.

10. Frokjaer JB, Bouwense SA, Olesen SS, Lundager FH, Eskildsen SF, van Goor $\mathrm{H}$, et al. Reduced cortical thickness of brain areas involved in pain processing in patients with chronic pancreatitis. Clin Gastroenterol Hepatol. 2012;10(4):434-8 e1.

11. Frokjaer JB, Olesen SS, Gram M, Yavarian Y, Bouwense SA, Wilder-Smith $\mathrm{OH}$, et al. Altered brain microstructure assessed by diffusion tensor imaging in patients with chronic pancreatitis. Gut. 2011;60(11):1554-62.

12. Lu C, Yang T, Zhao H, Zhang M, Meng F, Fu H, et al. Insular cortex is critical for the perception, modulation, and Chronification of pain. Neurosci Bull. 2016:32(2):191-201.

13. Zhuo M. Contribution of synaptic plasticity in the insular cortex to chronic pain. Neuroscience. 2016:338:220-9.

14. Qiu S, Zhang M, Liu Y, Guo Y, Zhao H, Song Q, et al. GluA1 phosphorylation contributes to postsynaptic amplification of neuropathic pain in the insular cortex. J Neurosci. 2014;34(40):13505-15.

15. Qiu S, Chen T, Koga K, Guo YY, Xu H, Song Q, et al. An increase in synaptic NMDA receptors in the insular cortex contributes to neuropathic pain. Sci Signal. 2013;6(275):ra34

16. Coffeen U, Manuel Ortega-Legaspi J, Lopez-Munoz FJ, Simon-Arceo K, Jaimes O, Pellicer F. Insular cortex lesion diminishes neuropathic and inflammatory pain-like behaviours. Eur J Pain (London, England). 2011; ](2):132-138.

17. Benison AM, Chumachenko S, Harrison JA, Maier SF, Falci SP, Watkins LR, et al. Caudal granular insular cortex is sufficient and necessary for the longterm maintenance of allodynic behavior in the rat attributable to mononeuropathy. J Neurosci. 2011;31(17):6317-28.

18. Jasmin L, Rabkin SD, Granato A, Boudah A, Ohara PT. Analgesia and hyperalgesia from GABA-mediated modulation of the cerebral cortex. Nature. 2003:424(6946):316-20.

19. Watson CJ. Insular balance of glutamatergic and GABAergic signaling modulates pain processing. Pain. 2016;157(10):2194-207.

20. Han J, Kwon M, Cha M, Tanioka M, Hong SK, Bai SJ, et al. Plasticity-related PKMzeta signaling in the insular cortex is involved in the modulation of neuropathic pain after nerve injury. Neural plasticity. 2015;2015:601767.

21. Zhang PA, Xu QY, Xue L, Zheng H, Yan J, Xiao Y, et al. Neonatal maternal deprivation enhances presynaptic $\mathrm{P} 2 \mathrm{X} 7$ receptor transmission in insular cortex in an adult rat model of visceral hypersensitivity. CNS Neurosci Ther. 2017;23(2):145-54

22. Zhang PA, Zhu HY, Xu QY, Du WJ, Hu S, Xu GY. Sensitization of P2X3 receptors in insular cortex contributes to visceral pain of adult rats with neonatal maternal deprivation. Mol Pain. 2018;14:1744806918764731.
23. Yi $L$, Sun $H$, Ge C, Chen $Y$, Peng $H$, Jiang $Y$, et al. Role of insular cortex in visceral hypersensitivity model in rats subjected to chronic stress. Psychiatry Res. 2014;220(3):1138-43.

24. Jongsma ML, Postma SA, Souren P, Arns M, Gordon E, Vissers K, et al. Neurodegenerative properties of chronic pain: cognitive decline in patients with chronic pancreatitis. PLoS One. 2011;6(8):e23363.

25. Madan A, Borckardt JJ, Barth KS, Romagnuolo J, Morgan KA, Adams DB. Interprofessional collaborative care reduces excess service utilization among individuals with chronic pancreatitis. J Healthc Qual. 2013;35(5):41-6.

26. Gasquoine PG. Contributions of the insula to cognition and emotion. Neuropsychol Rev. 2014;24(2):77-87.

27. Augustine JR. Circuitry and functional aspects of the insular lobe in primates including humans. Brain Res Brain Res Rev. 1996;22(3):229-44.

28. Barthas F, Sellmeijer J, Hugel S, Waltisperger E, Barrot M, Yalcin I. The anterior cingulate cortex is a critical hub for pain-induced depression. Biol Psychiatry. 2015;77(3):236-45

29. Craig AD. Interoception: the sense of the physiological condition of the body. Curr Opin Neurobiol. 2003;13(4):500-5

30. Zimmermann M. Ethical guidelines for investigations of experimental pain in conscious animals. Pain. 1983;16(2):109-10.

31. Winston JH, He ZJ, Shenoy M, Xiao SY, Pasricha PJ. Molecular and behavioral changes in nociception in a novel rat model of chronic pancreatitis for the study of pain. Pain. 2005;117(1-2):214-22.

32. Zhang MM, Liu SB, Chen T, Koga K, Zhang T, Li YQ, et al. Effects of NB001 and gabapentin on irritable bowel syndrome-induced behavioral anxiety and spontaneous pain. Mol Brain. 2014;7:47.

33. Wan FP, Bai Y, Kou ZZ, Zhang T, Li H, Wang YY, et al. Endomorphin-2 inhibition of substance $P$ signaling within Lamina I of the spinal cord is impaired in diabetic neuropathic pain rats. Front Mol Neurosci. 2016;9:167.

34. Mickle AD, Shepherd AJ, Loo L, Mohapatra DP. Induction of thermal and mechanical hypersensitivity by parathyroid hormone-related peptide through upregulation of TRPV1 function and trafficking. Pain. 2015;156(9):1620-36.

35. Chen T, Lu JS, Song Q, Liu MG, Koga K, Descalzi G, et al. Pharmacological rescue of cortical synaptic and network potentiation in a mouse model for fragile X syndrome. Neuropsychopharmacology. 2014;39(8):1955-67.

36. Majumder S, Chari ST. Chronic pancreatitis. Lancet. 2016;387(10031):1957-66

37. Coggeshall RE. Fos, nociception and the dorsal horn. Prog Neurobiol. 2005; 77(5):299-352.

38. Paxinos G WC. The Rat Brain in Stereotaxic Coordinates. 5th Edn. Amsterdam: Elsevier. 2005

39. Foster TC, McNaughton BL. Long-term enhancement of CA1 synaptic transmission is due to increased quantal size, not quantal content. Hippocampus. 1991;1(1):79-91.

40. Wojcik SM, Rhee JS, Herzog E, Sigler A, Jahn R, Takamori S, et al. An essential role for vesicular glutamate transporter 1 (VGLUT1) in postnatal development and control of quantal size. Proc Natl Acad Sci U S A. 2004; 101(18):7158-63.

41. Hollmann M, Hartley M, Heinemann S. Ca2+ permeability of KA-AMPAgated glutamate receptor channels depends on subunit composition. Science (New York, NY). 1991;252(5007):851-3.

42. Burnashev N, Schoepfer R, Monyer H, Ruppersberg JP, Gunther W, Seeburg $\mathrm{PH}$, et al. Control by asparagine residues of calcium permeability and magnesium blockade in the NMDA receptor. Science (New York, NY). 1992; 257(5075):1415-9.

43. Qiu S, Li XY, Zhuo M. Post-translational modification of NMDA receptor GluN2B subunit and its roles in chronic pain and memory. Semin Cell Dev Biol. 2011;22(5):521-9.

44. Zhang J, Abdullah JM. The role of GluA1 in central nervous system disorders. Rev Neurosci. 2013;24(5):499-505.

45. Lau CG, Zukin RS. NMDA receptor trafficking in synaptic plasticity and neuropsychiatric disorders. Nat Rev Neurosci. 2007;8(6):413-26.

46. Gerges NZ, Backos DS, Rupasinghe CN, Spaller MR, Esteban JA. Dual role of the exocyst in AMPA receptor targeting and insertion into the postsynaptic membrane. EMBO J. 2006:25(8):1623-34

47. Armbruster BN, Li X, Pausch MH, Herlitze S, Roth BL. Evolving the lock to fit the key to create a family of $G$ protein-coupled receptors potently activated by an inert ligand. Proc Natl Acad Sci U S A. 2007;104(12):5163-8.

48. Paulus MP, Stein MB. Interoception in anxiety and depression. Brain Struct Funct. 2010;214(5-6):451-63.

49. Ibanez A, Gleichgerrcht $E$, Manes F Clinical effects of insular damage in humans. Brain Struct Funct. 2010;214(5-6):397-410. 
50. Mayer EA, Gupta A, Kilpatrick LA, Hong JY. Imaging brain mechanisms in chronic visceral pain. Pain. 2015;156(Suppl 1):S50-63.

51. Liu MG, Chen J. Preclinical research on pain comorbidity with affective disorders and cognitive deficits: challenges and perspectives. Prog Neurobiol. 2014;116:13-32.

52. Felice VD, Moloney RD, Cryan JF, Dinan TG, O'Mahony SM. Visceral pain and psychiatric disorders. Mod Trends Pharmacopsychiatry. 2015;30:103-19.

53. Yoon MS, Koh CS, Lee J, Shin J, Kong C, Jung HH, et al. Injecting NMDA and Ro 25-6981 in insular cortex induce neuroplastic changes and neuropathic pain-like behaviour. Eur J Pain (London, England). 2018;22(9):1691-700.

54. Liu MG, Kang SJ, Shi TY, Koga K, Zhang MM, Collingridge GL, et al. Longterm potentiation of synaptic transmission in the adult mouse insular cortex: multielectrode array recordings. J Neurophysiol. 2013;110(2):505-21.

55. Nicoll RA, Schmitz D. Synaptic plasticity at hippocampal mossy fibre synapses. Nat Rev Neurosci. 2005;6(11):863-76.

56. Koga K, Descalzi G, Chen T, Ko HG, Lu J, Li S, et al. Coexistence of two forms of LTP in ACC provides a synaptic mechanism for the interactions between anxiety and chronic pain. Neuron. 2015;85(2):377-89.

57. Zhuo M. Neural mechanisms underlying anxiety-chronic pain interactions. Trends Neurosci. 2016;39(3):136-45.

58. Miao HH, Li XH, Chen QY, Zhuo M. Calcium-stimulated adenylyl cyclase subtype 1 is required for presynaptic long-term potentiation in the insular cortex of adult mice. Mol Pain. 2019;15:1744806919842961.

59. Winston JH, Toma H, Shenoy M, He ZJ, Zou L, Xiao SY, et al. Acute pancreatitis results in referred mechanical hypersensitivity and neuropeptide up-regulation that can be suppressed by the protein kinase inhibitor k252a. J Pain. 2003;4(6):329-37.

60. Talukdar R, Reddy DN. Pain in chronic pancreatitis: managing beyond the pancreatic duct. World J Gastroenterol. 2013;19(38):6319-28.

61. Lefaucheur JP. Cortical neurostimulation for neuropathic pain: state of the art and perspectives. Pain. 2016;157(Suppl 1):S81-9.

62. Ciampi de Andrade D, Galhardoni R, Pinto LF, Lancelotti R, Rosi J Jr, Marcolin MA, et al. Into the island: a new technique of non-invasive cortical stimulation of the insula. Neurophysiol Clin. 2012;42(6):363-8.

63. Deng ZD, Lisanby SH, Peterchev AV. Coil design considerations for deep transcranial magnetic stimulation. Clin Neurophysiol. 2014;125(6):1202-12.

64. Nguyen JP, Nizard J, Keravel Y, Lefaucheur JP. Invasive brain stimulation for the treatment of neuropathic pain. Nat Rev Neurol. 2011;7(12):699-709.

65. Plow EB, Pascual-Leone A, Machado A. Brain stimulation in the treatment of chronic neuropathic and non-cancerous pain. J Pain. 2012;13(5):411-24.

66. Lenoir C, Algoet M, Mouraux A. Deep continuous theta burst stimulation of the operculo-insular cortex selectively affects Adelta-fibre heat pain. J Physiol. 2018;596(19):4767-87.

67. Denis DJ, Marouf R, Rainville P, Bouthillier A, Nguyen DK. Effects of insular stimulation on thermal nociception. Eur J Pain (London, England). 2016; 20(5):800-10.

68. Dimov LF, Toniolo EF, Alonso-Matielo H, de Andrade DC, Garcia-Larrea L, Ballester $\mathrm{G}$, et al. Electrical stimulation of the insular cortex as a novel target for the relief of refractory pain: an experimental approach in rodents. Behav Brain Res. 2018;346:86-95.

69. Russo JF, Sheth SA. Deep brain stimulation of the dorsal anterior cingulate cortex for the treatment of chronic neuropathic pain. Neurosurg Focus. 2015;38(6):E11

\section{Publisher's Note}

Springer Nature remains neutral with regard to jurisdictional claims in published maps and institutional affiliations.

Ready to submit your research? Choose BMC and benefit from:
- fast, convenient online submission
- thorough peer review by experienced researchers in your field
- rapid publication on acceptance
- support for research data, including large and complex data types
- gold Open Access which fosters wider collaboration and increased citations
- maximum visibility for your research: over 100M website views per year
At BMC, research is always in progress.
Learn more biomedcentral.com/submissions

